\title{
Oxygen isotopic composition of chondritic interplanetary dust particles: A genetic link between carbonaceous chondrites and comets
}

\author{
J. Aléon ${ }^{\mathrm{a}, \mathrm{b}, *}$, C. Engrand ${ }^{\mathrm{c}, \mathrm{d}}$, L.A. Leshin ${ }^{\mathrm{c}, \mathrm{e}}$, K.D. McKeegan ${ }^{\mathrm{c}}$ \\ ${ }^{a}$ Lawrence Livermore National Laboratory, Glenn T. Seaborg Institute, P.O. Box 808, Livermore, CA 94550, USA \\ ${ }^{\mathrm{b}}$ Centre de Recherches Pétrographiques et Géochimiques, 15 rue Notre Dame des Pauvres BP 20, 54501 Vandoeuvre-les-Nancy, France \\ ${ }^{\mathrm{c}}$ Department of Earth and Space Sciences, University of California Los Angeles, 595 Charles Young Drive East, Los Angeles, \\ CA 90095-1567, USA \\ ${ }^{\mathrm{d}}$ Centre de Spectrométrie Nucléaire et de Spectrométrie de Masse, Bat 104, Orsay campus 91405, France \\ ${ }^{\mathrm{e}}$ NASA Goddard Space Flight Center, Greenbelt, MD 20771, USA
}

Received 5 September 2008; accepted in revised form 15 April 2009; available online 12 May 2009

\begin{abstract}
Oxygen isotopes were measured in four chondritic hydrated interplanetary dust particles (IDPs) and five chondritic anhydrous IDPs including two GEMS-rich particles (Glass embedded with metal and sulfides) by a combination of high precision and high lateral resolution ion microprobe techniques.

All IDPs have isotopic compositions tightly clustered around that of solar system planetary materials. Hydrated IDPs have mass-fractionated oxygen isotopic compositions similar to those of CI and CM carbonaceous chondrites, consistent with hydration of initially anhydrous protosolar dust. Anhydrous IDPs have small ${ }^{16} \mathrm{O}$ excesses and depletions similar to those of carbonaceous chondrites, the largest ${ }^{16} \mathrm{O}$ variations being hosted by the two GEMS-rich IDPs. Coarse-grained forsteritic olivine and enstatite in anhydrous IDPs are isotopically similar to their counterparts in comet Wild 2 and in chondrules suggesting a high temperature inner solar system origin. The small variations in the ${ }^{16} \mathrm{O}$ content of GEMS-rich IDPs suggest that most GEMS either do not preserve a record of interstellar processes or the initial interstellar dust is not ${ }^{16} \mathrm{O}$-rich as expected by self-shielding models, although a larger dataset is required to verify these conclusions.

Together with other chemical and mineralogical indicators, $\mathrm{O}$ isotopes show that the parent-bodies of carbonaceous chondrites, of chondritic IDPs, of most Antarctic micrometeorites, and comet Wild 2 belong to a single family of objects of carbonaceous chondrite chemical affinity as distinct from ordinary, enstatite, K- and R-chondrites. Comparison with astronomical observations thus suggests a chemical continuum of objects including main belt and outer solar system asteroids such as C-type, P-type and D-type asteroids, Trojans and Centaurs as well as short-period comets and other Kuiper Belt Objects.
\end{abstract}

(C) 2009 Elsevier Ltd. All rights reserved.

\footnotetext{
* Corresponding author. Present address: Centre de Spectrométrie Nucléaire et de Spectrométrie de Masse, Bat 104, Orsay campus 91405, France.

E-mail address: Jerome.Aleon@csnsm.in2p3.fr (J. Aléon).
}

\section{INTRODUCTION}

Oxygen isotope abundances in undifferentiated meteorites vary systematically relative to the Earth in a way that cannot be explained by classical mass fractionation from any specific starting composition (Clayton et al., 1973). The most pervasive large oxygen "isotopic anomalies" are the ${ }^{16} \mathrm{O}$ excesses (typically up to $5 \%$ ) observed in 
Calcium-Aluminum-rich Inclusions (CAIs), the oldest rocks that formed in the solar system (e.g. Amelin et al., 2002) by condensation at high temperature of the circumsolar gas (e.g. Grossman, 1972) followed by multiple heating events. Several models have been proposed to explain these observations: (1) injection of ${ }^{16} \mathrm{O}$ freshly synthesized in a supernova that could have triggered the collapse of the protosolar cloud (Clayton et al., 1973, 1977), (2) non-massdependent fractionation during condensation of the first solar system solids (Thiemens and Heidenreich, 1983; Thiemens, 1999; Marcus, 2004; Robert, 2004) or (3) isotope selective photodissociation of CO driven by UV irradiation in the presolar cloud (Yurimoto and Kuramoto, 2004), or in the protosolar accretion disk (Clayton, 2002; Lyons and Young, 2005). Deciphering the origin of these ${ }^{16} \mathrm{O}$ excesses and their distribution in solar system objects is thus of utmost importance to unravel the mechanisms of solar system formation and the origin of planetary materials.

These models and the dynamics of the solar nebula that they imply can be tested by analyzing the oxygen isotopic composition of several major reservoirs: (1) the Sun, which contains $99.8 \%$ of the mass of the solar system, (2) the gas from the inner regions of the nebula, (3) the dust from the inner regions of the nebula, (4) the gas from the outer regions of the nebula and (5) the dust from the outer solar nebula, i.e. mostly interstellar grains accreting onto the protosolar accretion disk. The oxygen isotopic composition of the Sun is the primary science goal of the Genesis mission. Two previous attempts to determine it from the solar wind implanted in metal from the lunar regolith have yielded contradictory results $\left({ }^{16} \mathrm{O}\right.$-rich, Hashizume and Chaussidon, 2005; ${ }^{16} \mathrm{O}$-poor, Ireland et al., 2006) probably because of multiple sources of oxygen on the lunar surface (Hashizume and Chaussidon, 2008). Preliminary results from the analysis of Genesis materials (McKeegan et al., 2009) indicate that the Sun is ${ }^{16} \mathrm{O}$-rich compared to planetary materials as predicted by some models (Clayton, 2002). The oxygen isotopic composition of the inner nebula gas and dust have been studied from numerous measurements of high temperature components of chondrites such as CAIs, their olivine-rich accretionary rims, and chondrules, and also of lower temperature components such as the hydrated minerals in CI and CM chondrites (Clayton and Mayeda, 1999; McKeegan and Leshin, 2001). It is currently believed that the first solar system solids (CAIs) condensed from a ${ }^{16} \mathrm{O}$-rich gas (Krot et al., 2002) and some of these reacted with a ${ }^{16} \mathrm{O}$-poor gas in which chondrules formed. The $\mathrm{O}$ isotopic composition of this gas is best recorded in chondrule mesostasis (Chaussidon et al., 2008). Hydrated matrix minerals formed by reaction of solids having a chondrulelike $\mathrm{O}$ isotopic composition with a water somewhat depleted in ${ }^{16} \mathrm{O}$ (e.g. Clayton and Mayeda, 1984, 1999; Choi et al., 1998; Young et al., 1999). The recent discovery of a large ${ }^{16} \mathrm{O}$ depletion in an oxidized metal phase, termed cosmic symplectite (COS), from the ungrouped C3 carbonaceous chondrite Acfer 094 (Sakamoto et al., 2007; Seto et al., 2008) suggests that early solar system water could have been very ${ }^{16} \mathrm{O}$-poor.

The oxygen isotopic composition of outer solar nebula gas and dust is still largely unknown. Hydrogen isotopes in the atmospheres of the giant planets are consistent with trapping of outer nebular gas and ices (e.g. Feuchtgruber et al., 1999; Lellouch et al., 2001), but the determination of oxygen isotopic compositions with a precision better than a few percent still requires laboratory analyses on Earth. One approach to determining the oxygen isotopic composition of outer nebular matter is from the analysis of cometary dust. Measurements of oxygen isotope abundances of dust from the Jupiter family comet Wild 2 returned to the Earth by the Stardust mission (Brownlee et al., 2006), reveals a striking similarity to the composition of high temperature grains (ferromagnesian silicates and $\mathrm{Ca}-\mathrm{Al}$-rich minerals) of comparable minerals in carbonaceous chondrites suggesting radial mixing at a large scale in the early solar system (McKeegan et al., 2006; Nakamura et al., 2008). However, most of the mass of Wild 2 dust collected by Stardust appears to be severely altered by heating during capture and/or mixed intimately with the silica aerogel collector medium (Zolensky et al., 2006). As a result, fragile components such as amorphous silicates of possible interstellar origin remain extremely difficult to analyze. Before the return of the Stardust samples, cometary samples could only be obtained from interplanetary dust in Earth crossing orbit. Such dust has been collected in the stratosphere (e.g. Brownlee, 1985) and is referred to as interplanetary dust particles (IDPs) and in the polar ices and snows, principally in Antarctica (e.g. Maurette et al., 1991; Duprat et al., 2007), and is referred to as Antarctic micrometeorites (AMMs).

Although comets must contribute significantly to the interplanetary dust population crossing $1 \mathrm{AU}$, the cometary origin of individual IDPs and AMMs is difficult to establish unambiguously. AMMs are usually larger than IDPs (up to several hundred $\mu \mathrm{m}$ ) and thus experienced more drastic modifications during atmospheric entry (e.g. Toppani et al., 2001; Nozaki et al., 2006). Nevertheless, the O isotopic composition of most AMMs is indicative of major similarities with carbonaceous chondrites: anhydrous ferromagnesian and refractory minerals have isotopic compositions comparable to that of chondrules and CAIs, respectively (Engrand et al., 1999a; Gounelle et al., 2005), although a significant fraction of coarse-grained AMMs may still be related to ordinary chondrites (Genge, 2006). In the case of stratosphere-collected IDPs, high precision $\mathrm{O}$ isotopic data allowing direct comparison with chondrites are limited to refractory particles, which have ${ }^{16} \mathrm{O}$ excesses typical of chondritic CAIs (McKeegan, 1987). Recent data, obtained with very high spatial resolution mapping in order to search for presolar interstellar grains, do not have sufficient precision to allow a direct comparison with chondrites (Messenger et al., 2003; Keller and Messenger, 2005).

Previous studies of stratospheric IDPs have shown that the properties of porous anhydrous particles have many similarities with those of cometary dust as inferred from astronomical observations and models: Mg-rich crystalline anhydrous silicates, amorphous silicates, high porosity, low density, mostly sub- $\mu \mathrm{m}$ grain size and high abundance of carbonaceous material. In addition, some anhydrous IDPs also have infrared spectra (Bradley et al., 1992) or elemental ratio distributions (Bradley, 1988) comparable with some 
comets. The mineralogical and petrological analysis of Stardust samples suggests that dust from comet Wild 2 is closest to these anhydrous IDPs than to any other meteoritic material (Zolensky et al., 2006), although it has also recently been claimed that comet Wild 2 may have more similarities with chondritic asteroids than with anhydrous IDPs (Ishii et al., 2008).

The purpose of this paper is to present a systematic high precision study of oxygen isotopic compositions of chondritic IDPs, including both hydrated and anhydrous particles and their subcomponents, in order to identify cometary dust, to determine its oxygen isotopic composition and to compare it with AMMs, carbonaceous chondrites and comet Wild 2. Preliminary results were reported by Engrand et al. (1999b) and Aléon et al. (2006).

\section{ANALYTICAL METHODS}

IDPs studied in this work were first characterized by Scanning Electron Microscopy (SEM) using standard and field-emission SEMs. Energy dispersive X-ray (EDX) spectra were taken to ensure the particles had chondritic abundances of major rock forming elements $(\mathrm{Si}, \mathrm{Mg}, \mathrm{Fe}, \mathrm{S}, \mathrm{Ca}$ and $\mathrm{Al}$ ). Secondary electron images were acquired before and after SIMS analysis of most particles to study their morphology and their state after SIMS analysis. X-ray maps were taken in IDPs not previously studied to characterize the elemental distribution and to estimate their mineralogy by comparison with the typical mineralogy of chondritic IDPs.

Oxygen isotope abundances were measured using the IMS 1270 ion microprobe of the University of California Los Angeles in two sessions under different analysis modes: (1) with a fixed defocused beam to determine the bulk $O$ isotopic composition of the particles with a $\sim 1-2 \%$ precision, hereafter termed "spot mode" analysis and (2) with a focused beam rastered over the sample in order to determine the $\mathrm{O}$ isotopic composition of selected components with a spatial resolution set by the beam size, hereafter referred to as "raster mode" analysis. The latter analyses have poorer precision, but it is still good enough to distinguish between the endmember compositions of meteoritic components (i.e. matrix, chondrules, CAIs).

\subsection{Spot mode analysis}

We used a primary $\mathrm{Cs}^{+}$beam in aperture illumination mode defocused to $10 \mu \mathrm{m}(0.2 \mathrm{nA})$ or $20 \mu \mathrm{m}(0.3 \mathrm{nA})$ in diameter and electron flooding for charge compensation. $\mathrm{O}$ isotopes were measured sequentially on a Faraday Cup $\left(\mathrm{FC},{ }^{16} \mathrm{O}\right)$ and an electron multiplier $\left(\mathrm{EM},{ }^{17} \mathrm{O},{ }^{18} \mathrm{O}\right)$ at a mass resolving power $>5000$ to resolve interference of ${ }^{16} \mathrm{OH}$ at mass 17 . Instrumental mass fractionation was determined from (1) polished sections of San Carlos and Eagle Station olivines and a Burma spinel and (2) grains of a San Carlos olivine crushed into Au foil in a manner similar to that of the IDPs. Data were corrected for FC background, EM deadtime, possible intensity drift due to the erosion of the IDPs and tail of the ${ }^{16} \mathrm{OH}$ peak $(<1 \%)$ on ${ }^{17} \mathrm{O}$. The overall error on IDPs analyzed in spot mode was typically $1-1.5 \%(1 \sigma)$. O isotope instrumental fractionations between silicates (matrix effects) depend on their $\mathrm{SiO}_{2}$ and $\mathrm{FeO}$ content (Leshin et al., 1997; Chaussidon et al., 2008). A higher $\mathrm{SiO}_{2}$ or $\mathrm{FeO}$ content relative to San Carlos olivine in our IDPs (e.g. phyllosilicates, pyroxene, amorphous silicates) would result in effects of at most $1-2 \%$, which remains comparable to our $1 \sigma$ analytical uncertainties. The largest possible effect not taken into account is a few $\%$ on pure magnetite. However, analyses of Fe-oxide-rich regions in IDP L2036R5 (see below) fall within the range of carbonaceous chondrite magnetite suggesting that potential matrix effects remained small and did not significantly affect the results.

\subsection{Raster mode analysis}

Analyses were performed with a $3 \mu \mathrm{m}$ focused $\mathrm{Cs}^{+}$beam of $5 \mathrm{pA}$. Although we used a low current, the beam density on the sample, when the raster size is close to the beam size, is comparable to that of a fixed beam analysis. Therefore use of electron flooding for charge compensation was required to obtain reproducible analyses of small grains despite the grains being embedded in gold, as verified by analyses of crushed grains of a San Carlos olivine. Because of the low ion current and of the large periphery/surface ratio of the small areas analyzed, analyses become extremely sensitive to surface contamination, especially when the raster size is several times larger than the beam size. Tests performed using the IMS 1270 ion microprobe at CRPG, Nancy, France using a $1.5 \mu \mathrm{m} \mathrm{Cs}{ }^{+}$beam and simultaneous detection of the three oxygen isotopes on electron multipliers showed that results are shifted by $\sim 10 \%$ toward surface contamination when the sample chamber vacuum is above $2 \times 10^{-9}$ Torr. The vacuum was thus kept below $2 \times 10^{-9}$ Torr during IDP analyses. All other conditions were similar to the spot mode analyses. The instrumental mass fractionation was corrected by interspersed analyses of crushed grains of San Carlos olivine mounted on a high purity gold foil. Raster analyses lasted $20 \mathrm{~min}$. The overall error on IDPs analyzed in raster mode was typically about $4-5 \%$ on $\delta^{17} \mathrm{O}(1 \sigma)$. In these conditions, all matrix effects are expected to remain well within analytical error.

\section{SAMPLE DESCRIPTION}

Eleven chondritic interplanetary dust particles were selected for oxygen isotope analysis. Several particles were previously studied for mineralogy (Bradley, 1988; Germani et al., 1990; Steele, 1990) or hydrogen and nitrogen isotopes (Aléon et al., 2001, 2003). Two of the IDPs previously analyzed were discarded; in one case a contaminant particle was found on top of the IDP (L2021K1) and the other was sputtered away before the end of the $\mathrm{O}$ isotope analyses resulting in very large error bars (L2021A6). Nine chondritic IDPs were thus available for the study (Table 1). Initial NASA designations for two particles (GM4-2 and PP67) were lost before the particles were made available to us. A literature search for the previously studied IDP "GM4-2" yielded conflicting results. Indeed, GM4-2 was initially thought to be particle U222C6 from NASA 
Table 1

Oxygen isotopes in chondritic interplanetary dust particles.

\begin{tabular}{|c|c|c|c|c|c|c|}
\hline IDP & Mineralogy $^{\mathrm{a}}$ & Analysis & Properties $^{\mathrm{a}}$ & $\delta^{17} \mathrm{O}(\% \mathrm{oo})^{\mathrm{b}}$ & $\delta^{18} \mathrm{O}(\%)^{\mathrm{b}}$ & $\left.\Delta^{17} \mathrm{O}(\%)\right)^{\mathrm{b}}$ \\
\hline \multicolumn{7}{|l|}{ Hydrated } \\
\hline U222C29 & cpct, Mg-smect, carb, sulf & Spot 1 & Bulk & $6.9 \pm 2.8$ & $13.2 \pm 3.0$ & $0.0 \pm 3.2$ \\
\hline PP67 & cpct, Mg-sil, met, sulf & Spot 1 & Depth 1 & $9.3 \pm 2.2$ & $10.6 \pm 2.8$ & $3.8 \pm 2.6$ \\
\hline PP67 & cpct, Mg-sil, met, sulf & Spot 2 & Depth 2 & $4.9 \pm 2.4$ & $10.2 \pm 2.6$ & $-0.4 \pm 2.8$ \\
\hline PP67 & cpct, Mg-sil, met, sulf & Spot 3 & Depth 3 & $2.7 \pm 2.6$ & $7.6 \pm 3.0$ & $-1.3 \pm 3.0$ \\
\hline L2036R5 & cpct, Mg-sil, Fe-frb & Spot 1 & Area 1 & $4.0 \pm 1.4$ & $3.8 \pm 0.8$ & $2.0 \pm 1.4$ \\
\hline L2036R5 & cpct, Mg-sil, Fe-frb & Spot 2 & Area 2 & $7.8 \pm 1.0$ & $17.1 \pm 1.2$ & $-1.1 \pm 1.2$ \\
\hline L2036R5 & cpct, Mg-sil, Fe-frb & Spot 3 & Area 3 & $6.4 \pm 1.6$ & $8.3 \pm 1.2$ & $2.1 \pm 1.6$ \\
\hline L2036B3 & cpct, Mg-sil, Fe-frb & Spot 1 & Bulk & $12.7 \pm 2.0$ & $24.6 \pm 1.6$ & $-0.1 \pm 2.2$ \\
\hline L2036B3 & cpct, Mg-sil, Fe-frb & Raster $32 \mu \mathrm{m}$ & Bulk & $17.5 \pm 7.8$ & $27.8 \pm 5.2$ & $3.0 \pm 8.2$ \\
\hline L2036B3 & cpct, Mg-sil, Fe-frb & Raster $8 \mu \mathrm{m}$ & Fe-rich & $20.4 \pm 7.8$ & $34.5 \pm 4.2$ & $2.4 \pm 8.0$ \\
\hline \multicolumn{7}{|l|}{ Anhydrous } \\
\hline GM4-2 & fg, GEMS-rich & Spot 1 & Bulk & $-19.5 \pm 3.8$ & $-19.7 \pm 3.2$ & $-9.3 \pm 4.2$ \\
\hline L2036W1 & fg, GEMS-rich & Spot 1 & Bulk & $6.7 \pm 2.4$ & $7.1 \pm 1.4$ & $3.0 \pm 2.6$ \\
\hline U211A19A & cg En + Fo, fg crust & Spot 1 & En & $-3.5 \pm 2.4$ & $-1.6 \pm 2.6$ & $-2.7 \pm 2.8$ \\
\hline U211A19A & cg En + Fo, fg crust & Spot 2 & $\mathrm{Fo}_{98}$ & $-2.2 \pm 2.2$ & $0.0 \pm 2.6$ & $-2.2 \pm 2.6$ \\
\hline U211A19A & cg En + Fo, fg crust & Spot 3 & En & $-3.9 \pm 1.8$ & $-2.9 \pm 1.6$ & $-2.4 \pm 2.0$ \\
\hline RB27A19 & cg LIME - Fo, fg crust & Spot 1 & LIME - Fo ${ }_{97.5}$ & $-1.3 \pm 1.8$ & $1.0 \pm 2.0$ & $-1.8 \pm 2.2$ \\
\hline L2021N3 & $\operatorname{cg} \mathrm{Fo}+\mathrm{fg} \mathrm{mtx}$ & Spot 1 & Bulk & $-2.2 \pm 2.8$ & $0.5 \pm 2.8$ & $-2.4 \pm 3.2$ \\
\hline L2021N3 & $\operatorname{cg~Fo}+f g m t x$ & Raster $11 \mu \mathrm{m}$ & Bulk $-\mathrm{Fo}^{\mathrm{c}}$ & $13.9 \pm 12.2$ & $6.9 \pm 6.4$ & $10.4 \pm 12.6$ \\
\hline L2021N3 & $\operatorname{cg} F o+f g m t x$ & Raster $7 \mu \mathrm{m}$ & $\mathrm{Fo}_{\mathrm{O}}>85$ & $-1.1 \pm 10.4$ & $-1.0 \pm 5.6$ & $-0.6 \pm 10.8$ \\
\hline
\end{tabular}

a Abbreviations: cpct - compact; smect - smectite; carb - carbonate; sulf - sulfide; met - metal; sil - silicate; frb - framboids; fg - finegrained; cg - coarse-grained; En - enstatite; Fo - forsterite; LIME - low-iron-manganese-enriched; mtx - matrix; GEMS - glass with embedded metal and sulfides.

${ }^{\mathrm{b}}$ Error bars are $2 \sigma$ standard errors of the mean.

${ }^{c}$ Area containing most of the particle with the exception of the big olivine grain.

catalogs (Engrand et al., 1999b). However, Bradley (1988) indicates that U222C6 is a smectite-rich hydrated IDP, whereas GM4-2 is actually an anhydrous IDP (Engrand et al., 1999b) rich in nodules of Glass with embedded metal and sulfides (GEMS). For purposes of this paper, we refer to this IDP simply as GM4-2 and base no inference on the previous work by Bradley (1988) which appears to have been on a different sample.

In contrast to the situation for micrometeorites (Genge et al., 2008), there is no clear classification of chondritic IDPs. Here we use a simplified classification in two main groups, dominantly hydrated IDPs and dominantly anhydrous IDPs, keeping in mind that anhydrous minerals can be present in hydrated objects (e.g. olivine or pyroxene in CI chondrites, chondrules in CM chondrites) and vice-versa. IDPs are classified here as hydrated based on mineralogy when available or on chemistry (e.g. low $\mathrm{C} / \mathrm{H}$ ratios). This classification typically encompasses that of particles commonly termed chondritic smooth (CS, Brownlee, 1985). For this reason, particles with smooth morphologies (fine-grained compact) are also included in this group. Anhydrous particles include here fined-grained fluffy particles also termed chondritic porous anhydrous (CPA, Brownlee, 1985), but also coarse-grained olivine or pyroxene particles analogous to the crystalline AMM category (Genge et al., 2008 ) or to chondrule-like objects in Wild 2 (Nakamura et al., 2008), notably when CPA material is adhering to the crystals. This simple classification is also chosen to facilitate the $\mathrm{O}$ isotope comparison with carbonaceous chondrites (CCs), since anhydrous minerals in CCs commonly plot on a slope $\sim 1$ line in the three oxygen isotope diagram, whereas hydrated minerals show evidence of mass fractionation associated with hydration. Finally, several samples analyzed are derived from cluster particles (hydrated IDP L2036B3 and anhydrous IDPs L2036W1 and L2036N3), but this is information is not known for several other IDPs (hydrated IDPs U222C29 and PP67 and anhydrous IDPs GM4-2, U211A19A and RB27A19).

\subsection{Hydrated IDPs}

$\mathrm{U} 222 \mathrm{C} 29$ is a $\sim 7 \mu \mathrm{m}$ compact smectite-rich particle previously studied by transmission electron microscopy (Germani et al., 1990). It contains up to $2 \mu \mathrm{m} \mathrm{Mg-Fe-rich}$ carbonates embedded in a saponite matrix with minor diopside, pyrrhotite, enstatite, troilite, graphitized carbon and secondary magnetite. $\mathrm{O}$ isotopes were measured on fragments of the particle remaining in the epoxy bullet initially used for ultramicrotomy. One analysis with a $10 \mu \mathrm{m}$ spot was obtained on the particle.

PP67 is a $\sim 10 \mu \mathrm{m}$ compact particle dominated by $\mathrm{Mg}$ rich silicates. X-ray maps revealed that it contains FeNi metal grains and Ni-rich Fe-sulfides. Sub- $\mu \mathrm{m} \mathrm{Ca-Al-rich}$ phases are also present. Its compact morphology and the presence of significant $\mathrm{Ni}$ in sulfides is typical of hydrated IDPs. The particle was embedded in epoxy and polished before analysis. Three analyses with a $10 \mu \mathrm{m}$ spot were 
repeatedly obtained on the particle, giving an estimate of the isotopic heterogeneity in depth.

L2036R 5 is a large $(\sim 70 \mu \mathrm{m}$ after crushing $)$ compact IDP dominated by sub- $\mu \mathrm{m}$ grains. Carbon and hydrogen ion mapping have shown that the particle is dominantly hydrated $\left(\mathrm{C} / \mathrm{H}_{\text {bulk }} \sim 0.25\right)$ and has chondritic $\mathrm{D} / \mathrm{H}$ ratio $(\mathrm{D} /$ $\mathrm{H}_{\text {bulk }}=1.3 \times 10^{-4}$; Aléon et al., 2001). EDX maps show that it is dominated by Mg-rich silicates, probably phyllosilicates, but that several areas of Fe-rich oxides with framboidal textures are also present (Fig. 1). The framboid morphology of these Fe-oxides is typical of magnetite precipitated from a fluid as observed in CI and CR chondrites (e.g. Jedwab, 1971) and is different from that of magnetite rims formed during atmospheric entry heating, either as nanocrystals (IDPs, e.g. Fraundorf, 1981; Thomas et al., 1995), skeletal crystals (AMMs, Toppani et al., 2001) or euhedral crystals embedded in silicate glass (AMMs, Toppani et al., 2001). In addition, similar magnetite framboids were previously reported in hydrated IDPs attributed to a
CI parent body (e.g. Brownlee, 1985). The particle was pressed onto a high purity gold foil and three analyses with a $20 \mu \mathrm{m}$ spot were acquired in several areas, giving an estimate of the lateral isotopic heterogeneity.

L2036B3 is a $\sim 10 \mu \mathrm{m}$ similarly fine-grained compact IDP. Distribution of $\mathrm{Mg}, \mathrm{Si}$ and Fe in EDX maps are comparable to those of L2036R5. Framboids are also associated with Fe-rich regions suggesting the presence of magnetite. Being similar to L2036R5, it is also probably a hydrated IDP. The particle was pressed onto a high purity $\mathrm{Au}$ foil ( $\sim 25 \mu \mathrm{m}$ after crushing) and was analyzed using (1) a $20 \mu \mathrm{m}$ spot, (2) a $3 \mu \mathrm{m}$ spot rastered over the whole particle $(32 \mu \mathrm{m})$ and (3) a $3 \mu \mathrm{m}$ spot rastered over the magnetite-rich area $(8 \mu \mathrm{m})$.

\subsection{Anhydrous IDPs}

GM4-2 is a $\sim 10 \mu \mathrm{m}$ GEMS-rich porous particle with Fesulfides, FeMg carbonates and a $\mathrm{Cr}$-oxide grain. $\mathrm{O}$ isotopes

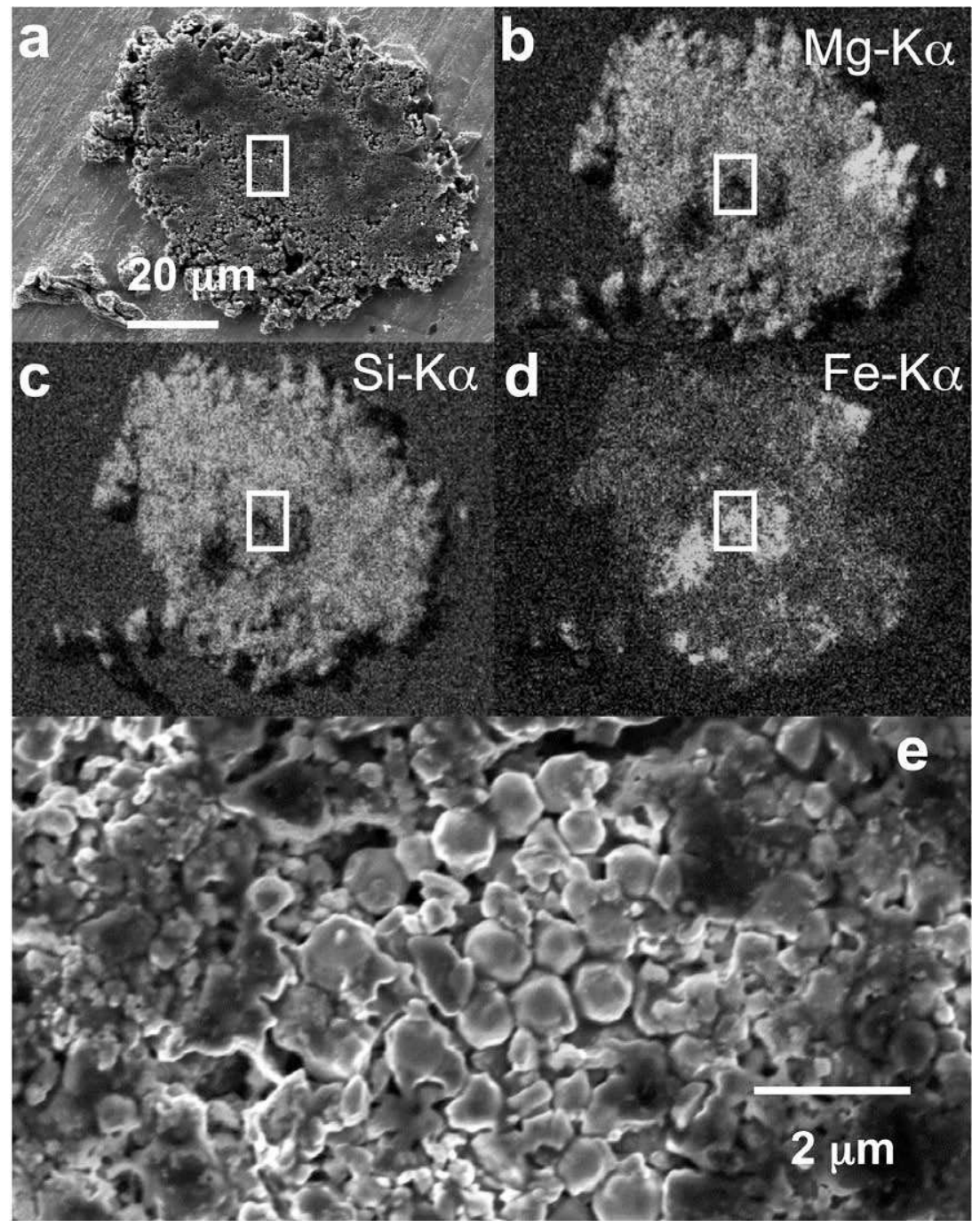

Fig. 1. Electron microscopy of hydrated IDP L2036R5. The white square in panels a, b, c and d indicates the location of panel e. (a) Secondary electrons (SE) image of the whole IDP after crushing into a high purity gold foil. (b-d) X-ray maps of $\mathrm{Mg}, \mathrm{Si}$ and Fe, respectively, indicating that the particle is dominated by Mg-silicates. (e) High resolution SE image of the framboidal texture of the central Fe-rich region suggesting the presence of magnetite. 
were measured on the remainder of the particle mounted in the epoxy bullet initially used for ultramicrotomy. One analysis with a $10 \mu \mathrm{m}$ spot was obtained on the particle.

L2036W1 is a $7 \times 5 \mu \mathrm{m}$ IDP consisting only of sub- $\mu \mathrm{m}$ grains. EDX maps showed a homogeneous distribution of $\mathrm{Si}, \mathrm{Mg}$ and $\mathrm{Fe}$, indicating that the particle is chondritic at the scale of the EDX analysis volume $\left(\sim 1 \mu \mathrm{m}^{3}\right)$. Mixed secondary-backscattered electron imaging with a field-emission SEM (5 keV) after crushing and ion probe analysis shows that all grains are between $100 \mathrm{~nm}$ and $500 \mathrm{~nm}$ in size with $30-50 \%$ of the grains containing bright (i.e. high-Z) rounded nanoinclusions comparable in size, shape and size distribution to metal and sulfides inclusions in GEMS (Fig. 2). Formation of nanoparticles with GEMS-like morphology upon irradiation by the ion probe beam can be excluded because (1) this effect has never been observed in ion probe crater pits to our knowledge despite hundreds of SEM observations after SIMS analysis, (2) we did not observe it in the other IDP samples imaged by high resolution SEM and (3) other transmission electron microscopy studies of IDPs after SIMS analysis have shown unmodified GEMS and did not reveal evidence of nanoparticle formation (e.g. Floss et al., 2004). Because this morphology is unique to GEMS in IDPs, these grains are probably GEMS although no detailed nanoscale mineralogical investigation was carried out. This probably GEMS-rich IDP was crushed into a high purity gold foil and analyzed using a $20 \mu \mathrm{m}$ spot.

U211A19A is a large $(40 \times 60 \mu \mathrm{m})$ IDP consisting mainly of coarse-grained $(20-40 \mu \mathrm{m})$ enstatite intergrown with a $15 \mu \mathrm{m}$ forsterite grain $\left(\mathrm{Fo}_{98}\right)$ and encased in sub$\mu \mathrm{m}$ porous chondritic material including $\mathrm{FeNi}$-sulfide. It has previously been referred to as a chondritic porous IDP with unusually large crystals (Steele, 1990). Manganese content of the forsterite is at the upper limit of the typical meteoritic range $(\mathrm{MnO} \sim 0.43 \mathrm{wt} \%$, Steele, 1990). Three O isotope analyses were acquired in enstatite and forsterite with a $10 \mu \mathrm{m}$ spot on a polished section previously prepared for electron microprobe analyses (Steele, 1990).

$\mathrm{RB} 27 \mathrm{~A} 19$ is a $10 \times 20 \mu \mathrm{m}$ particle consisting mostly of coarse-grained $(>10 \mu \mathrm{m})$ low-iron-manganese-enriched forsterite (LIME, Fo $97.5, \mathrm{MnO} \sim 0.82 \mathrm{wt}^{\%} \%$ ) surrounded by a crust of sub- $\mu \mathrm{m}$ porous chondritic material containing FeNi-sulfides. Together with U211A19A, it has previously been referred to as a chondritic porous IDP with unusually large crystals (Steele, 1990). O isotopes were measured in forsterite with a $10 \mu \mathrm{m}$ spot on a polished section prepared for electron microprobe analyses (Steele, 1990).

L $2021 \mathrm{~N} 3$ is a $10 \times 5 \mu \mathrm{m}$ IDP consisting of relatively coarse grains $(1-5 \mu \mathrm{m})$ embedded in a sub- $\mu \mathrm{m}$ porous matrix (Fig. 3). EDX maps and analyses reveal that the coarser grains are: (1) a $\sim 5 \mu \mathrm{m}$, roughly euhedral, forsteritic olivine with $\mathrm{Mg}$ content higher than a neighboring San Carlos olivine crushed grain ( $\mathrm{Fo}_{85-90}$ in San Carlos), (2) a $2 \mu \mathrm{m}$ anorthite, (3) three $1 \mu \mathrm{m}$ Fe-rich grains, and (4) possibly two $1 \mu \mathrm{m}$ diopside grains although in the latter case contamination by the surrounding fine-grained matrix prevents conclusive identification. SEM imaging was done in conditions similar to L2036W1 but no evidence of GEMS was found. The particle was pressed onto a high purity Au foil and was analyzed using: (1) a $20 \mu \mathrm{m}$ spot, (2) a $3 \mu \mathrm{m}$ spot rastered over the $5 \mu \mathrm{m}$ forsterite ( $7 \mu \mathrm{m}$ raster). The anorthite (an) crystal may represent a minor contribution (at most a few $\%$ ) in the



Fig. 2. Electron microscopy of anhydrous IDP L2021N3. (a) Secondary electrons (SE) image of the particle before crushing showing that it is dominated by fine-grained submicron material with an embedded $5 \mu \mathrm{m}$ forsteritic olivine (Fo) crystal. (b-f) X-ray maps of $\mathrm{Si}, \mathrm{Mg}, \mathrm{Fe}, \mathrm{Al}$ and $\mathrm{Ca}$, respectively, after crushing of the particle into high purity gold. Several coarse grains are visible embedded in a fine-grained $\mathrm{Mg}$-silicate matrix: the large Fo crystal, a Ca-Al-rich silicate, which is possibly anorthite (An) and three Fe-rich grains (arrows) depleted in $\mathrm{Si}$ and $\mathrm{Mg}$ (probably metal or sulfides). (g) SE image of the particle after crushing with the two raster analyses indicated (11 $\mu \mathrm{m}$ - mostly the fine-grained portion and $7 \mu \mathrm{m}-$ mostly Fo). 


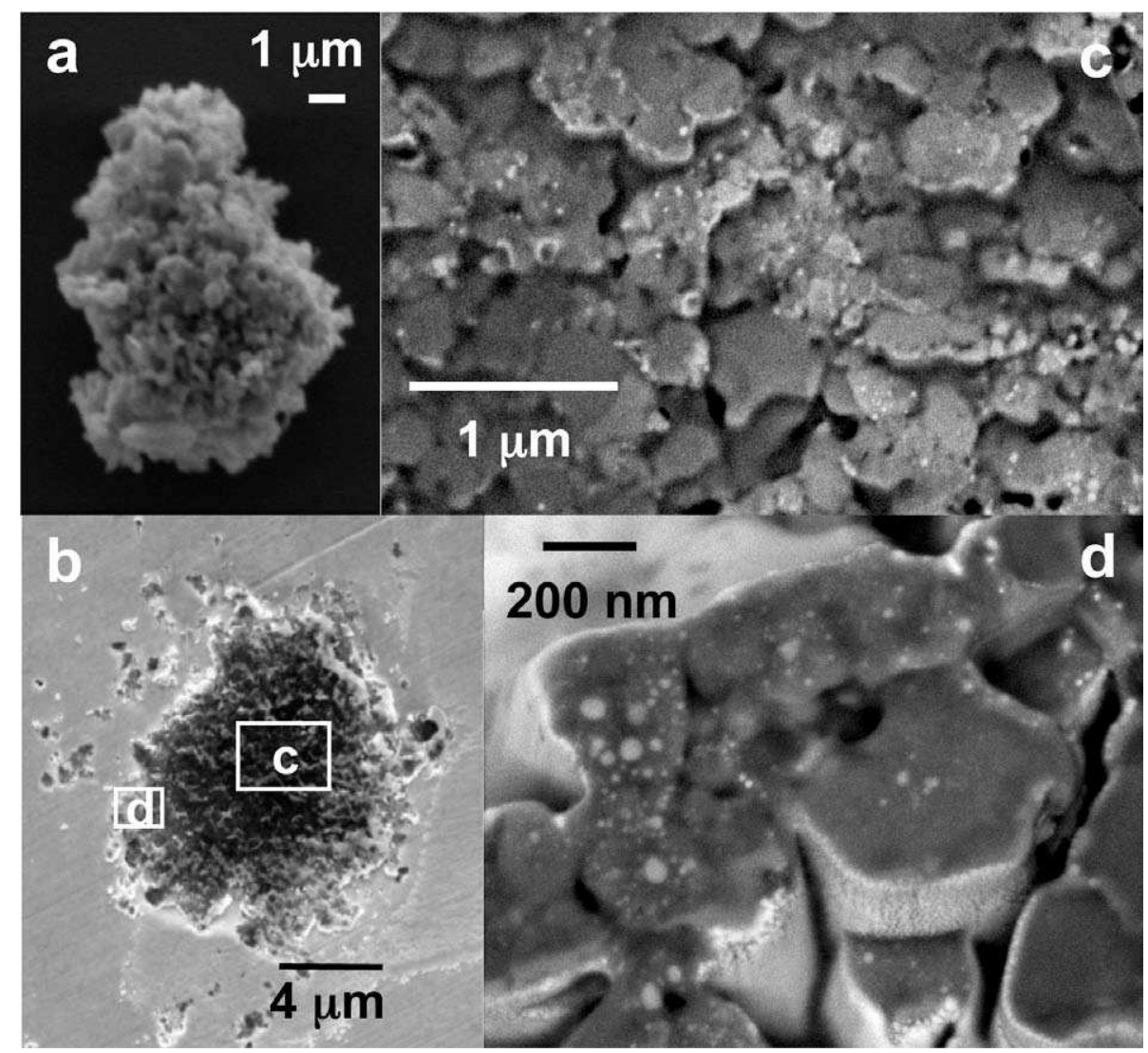

Fig. 3. Electron microscopy of GEMS-rich IDP L2036W1. (a) Secondary electron (SE) image of the particle before crushing. (b) SE image of the particle after crushing into high purity gold with locations of panels $\mathrm{c}$ and $\mathrm{d}$ indicated. $\mathrm{a}$ and $\mathrm{b}$ show that the IDP is only composed of submicron grains. (c) High resolution mixed SE-BSE (backscattered electrons) image after ion probe sputtering revealing that many submicron grains include abundant bright (i.e. heavy) nanometer-sized inclusions. (d) High resolution mixed SE-BSE image showing that the size and geometry of the nanoinclusions-rich grains, on one hand, and the distribution and sizes of their nanoinclusions, on the other hand, are comparable to those of GEMS and their metal and sulfides nanoinclusions, respectively, as studied by transmission electron microscopy (e.g. Bradley, 1994). Because only GEMS contain such nanoinclusions in IDPs, this indicates that the inclusion-containing grains are in fact GEMS.

latter analysis but the isotopic effect will remain undetectable, even if the isotopic composition of anorthite differs from that of the olivine grain by $50 \%$. Finally, (3) a $3 \mu \mathrm{m}$ spot was rastered over the bulk of the particle sitting next to the forsterite grain $(11 \mu \mathrm{m}$ raster $)$.

\section{RESULTS}

Oxygen isotope results are reported as permil deviations from the Standard Mean Ocean Water $\left(\delta^{18} \mathrm{O}=\right.$ $\left(\left[{ }^{18} \mathrm{O} /{ }^{16} \mathrm{O}\right]_{\text {sple }} /\left[{ }^{18} \mathrm{O} /{ }^{16} \mathrm{O}\right]_{\text {SMOw }}-1\right) \times 1000$, similarly for $\left.\delta^{17} \mathrm{O}\right) .{ }^{16} \mathrm{O}$ excesses or depletions are reported as deviations from the terrestrial mass fractionation line $\left(\Delta^{17} \mathrm{O}\right.$ $\left.=\delta^{17} \mathrm{O}-0.52 \times \delta^{18} \mathrm{O}\right)$. Errors are $2 \sigma$ standard error of the mean (95\% level of confidence) and include in-run uncertainties and external reproducibility on the standards.

\subsection{All samples}

Based on assumptions of their respective origins, it is expected that chondritic IDPs might show two highly distinct types of $\mathrm{O}$ isotopic compositions. Hydrated IDPs, for which an asteroidal origin has been proposed (e.g. Bradley and Brownlee, 1986), could have isotopic compositions analogous to those of hydrated carbonaceous chondrites. In contrast, anhydrous IDPs are thought to derive from mineralogically primitive objects (e.g. Bradley and Brownlee, 1986) potentially rich in interstellar dust grains and thus they might be expected to consist of heterogeneous, highly isotopically anomalous sub- $\mu \mathrm{m}$ components. Recent high spatial resolution $\mathrm{O}$ isotopic mapping of anhydrous IDPs by NanoSIMS (Keller and Messenger, 2005) indicates that sub- $\mu \mathrm{m}$ grains with isotopic anomalies larger than $10 \%$ are rare ( $\sim 1000$ ppm maximum), however, these relatively low precision and self-normalized data cannot address the existence of isotopic anomalies in the several $\%$ range similar to those observed in individual components of chondritic meteorites.

Oxygen isotopes in all samples fall in the range of typical planetary materials $\left(-9.3 \pm 4.2 \%<\Delta^{17} \mathrm{O}<10.4 \pm 12.6 \%\right.$, Table 1). Most of the chondritic IDPs show little or no deviation from the terrestrial mass fractionation line (TF line), with a total range similar to that of the bulk of 
carbonaceous chondrites. Although GM4-2 shows evidence for an ${ }^{16} \mathrm{O}$ excess, no chondritic IDP exhibits the typical ${ }^{16} \mathrm{O}$-rich composition seen in refractory inclusions, nor the large ${ }^{16} \mathrm{O}$ depletion recently found in a hydrated phase from the ungrouped C3 chondrite Acfer 094 (Sakamoto et al., 2007). Finally, no IDP shows evidence at the $>7 \mu \mathrm{m}$ scale of large isotopic anomalies attributable to stellar nucleosynthesis (e.g. Messenger et al., 2003), in agreement with statistical estimates from NanoSIMS mapping (Keller and Messenger, 2005).

\subsection{Hydrated IDPs}

Chondritic hydrated IDPs (Fig. 4, Table 1) have oxygen isotopic compositions close to the TF line and show evidence for mass fractionation with $\delta^{18} \mathrm{O}$ ranging from $3.8 \pm 0.8 \%$ in L2036R 5 to values comparable to carbonates in hydrated carbonaceous chondrites $\left(\delta^{18} \mathrm{O}=34.5 \pm 4.2 \%\right.$ in L2036B3). Isotopic heterogeneities are observed in single particles in both the 1998 and 2005 analyses, either laterally (e.g. $\delta^{18} \mathrm{O}$ varying from $3.8 \pm 0.8 \%$ to $17.1 \pm 1.2 \%$ in L2036R5) or with depth (e.g. $\Delta^{17} \mathrm{O}$ varying from $-1.3 \pm 3.0 \%$ to $3.8 \pm 2.6 \%$ in PP67). The Fe-rich area in L2036B3 is not statistically different from the bulk particle although it could be heavier by $\sim 3-5 \%$ o $/ \mathrm{amu}$ at the $68 \%$ $(1 \sigma)$ level of confidence (Table 1$)$.

\subsection{Anhydrous IDPs}

Oxygen isotopes in anhydrous IDPs do not show evidence for mass fractionation, in contrast to hydrated IDPs, but they do show a variation in ${ }^{16} \mathrm{O}$ content (Fig. 5). Although most IDPs are close to the terrestrial values, GEMS-rich IDPs show the largest range of $\Delta^{17} \mathrm{O}$ values, from $-9.3 \pm 4.2 \%$ in GM4-2 to $3.0 \pm 2.6 \%$ in L $2036 \mathrm{~W} 1$. Similarly, although no GEMS have been conclusively identified, the fine-grained portion of L2021N3 that contains a $2 \mu \mathrm{m}$ anorthite grain and possibly diopside is potentially depleted in ${ }^{16} \mathrm{O}$ by $\sim 10 \%$ but is undistinguishable from the bulk (or the TF line) at the $95 \%$ confidence level. Coarsegrained forsterite and enstatite, including the individual $5 \mu \mathrm{m}$ olivine from L2021N3 and the Mn-rich forsterite grains from U211A19A and RB27A19, have a very restricted range of oxygen isotopic compositions with $\Delta^{17} \mathrm{O}$ values clustering around $-2 \%$. Oxygen isotopes in anhydrous IDPs thus plot along a line a slope $1.08 \pm 0.14$ with an intercept at $-2.73 \pm 1.05$, which is comparable to the slope 1.0 line defined in unaltered portions of a CAI $\left(\delta^{17} \mathrm{O}=1.0 \times \delta^{18} \mathrm{O}-1.04\right.$; Young and Russell, 1998) but the data also remain within error of the CCAM line (Carbonaceous Chondrite Anhydrous Minerals mixing line, $\delta^{17} \mathrm{O}=0.94 \times \delta^{18} \mathrm{O}-4.5$; Clayton and Mayeda, 1984) defined from many CAIs and chondrules in CV chondrites.

\section{DISCUSSION}

Oxygen isotopes have been used as a classification tool to investigate the genetic relationships between meteorites and to define meteorite families (e.g. Clayton et al., 1991; Clayton and Mayeda, 1996, 1999). In a similar way, micro-
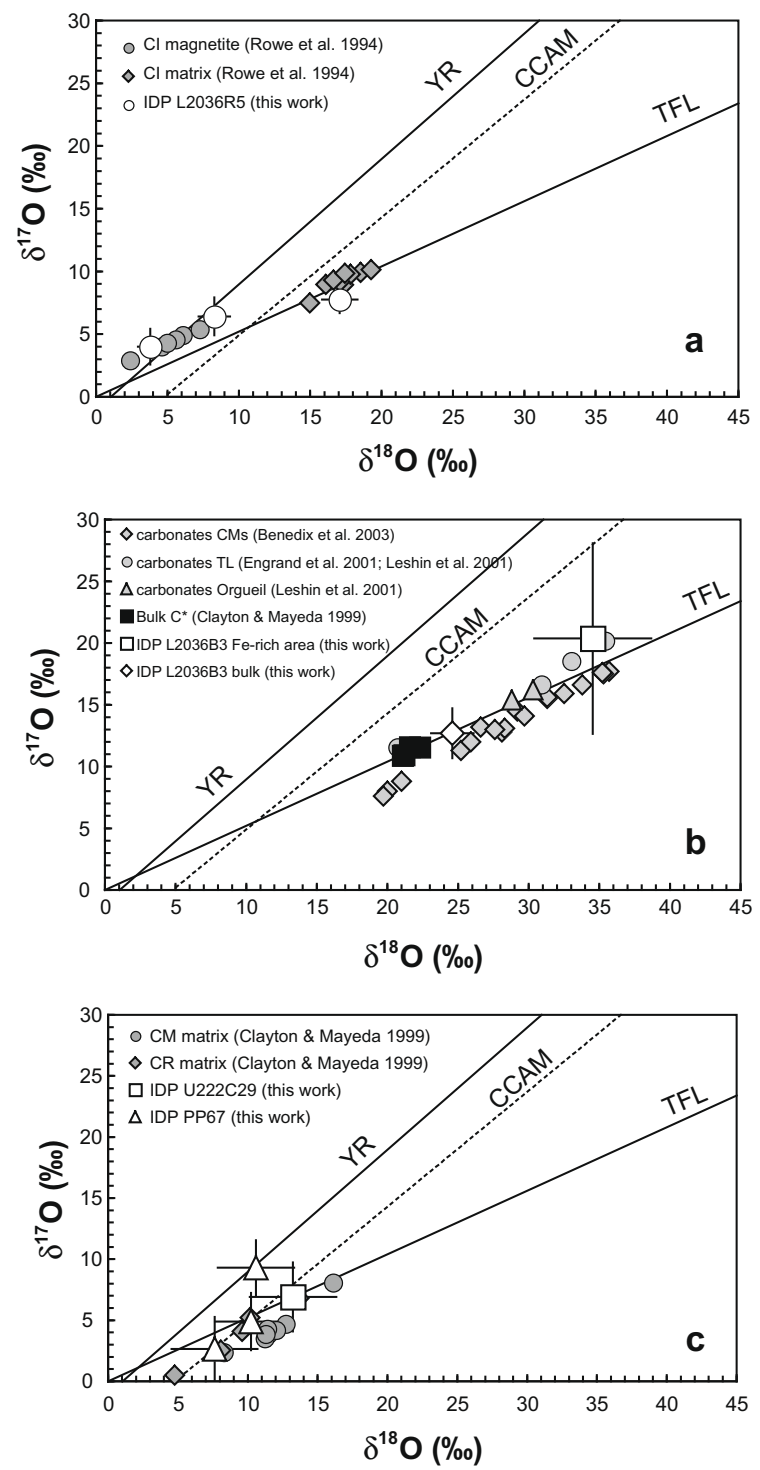

Fig. 4. O isotopes in hydrated IDPs. (a) IDP L2036R5 compared with magnetite and phyllosilicates from CI chondrites showing that L2035R 5 probably originated from a CI parent body. (b) L2036B3 compared with bulk $\mathrm{CI}$ and $\mathrm{C} *$ chondrites (see text) and with carbonates from Orgueil, Tagish Lake and CM chondrites. (c) $\mathrm{U} 222 \mathrm{C} 29$ and PP67 compared with matrices of $\mathrm{CM}$ and $\mathrm{CR}$ chondrites. Errors bars are $2 \sigma$ (not shown for literature data). TFL stands for Terrestrial mass Fractionation Line, CCAM stands for Carbonaceous Chondrite Anhydrous Minerals mixing line and YR stands for Young and Russell slope 1.0 line (Young and Russell, 1998).

meteorites have been shown to be related to carbonaceous chondrites on the basis of the $\mathrm{O}$ isotopic compositions of their anhydrous minerals (Engrand et al., 1999a; Gounelle et al., 2005). However, IDPs have never been classified nor compared to meteorites with respect to $\mathrm{O}$ isotopes before this study. In the following, we extend the $\mathrm{O}$ isotope classification of extraterrestrial materials to primitive dust potentially from outer solar system objects and discuss the nature of IDP parent-bodies before using the $\mathrm{O}$ isotopic 

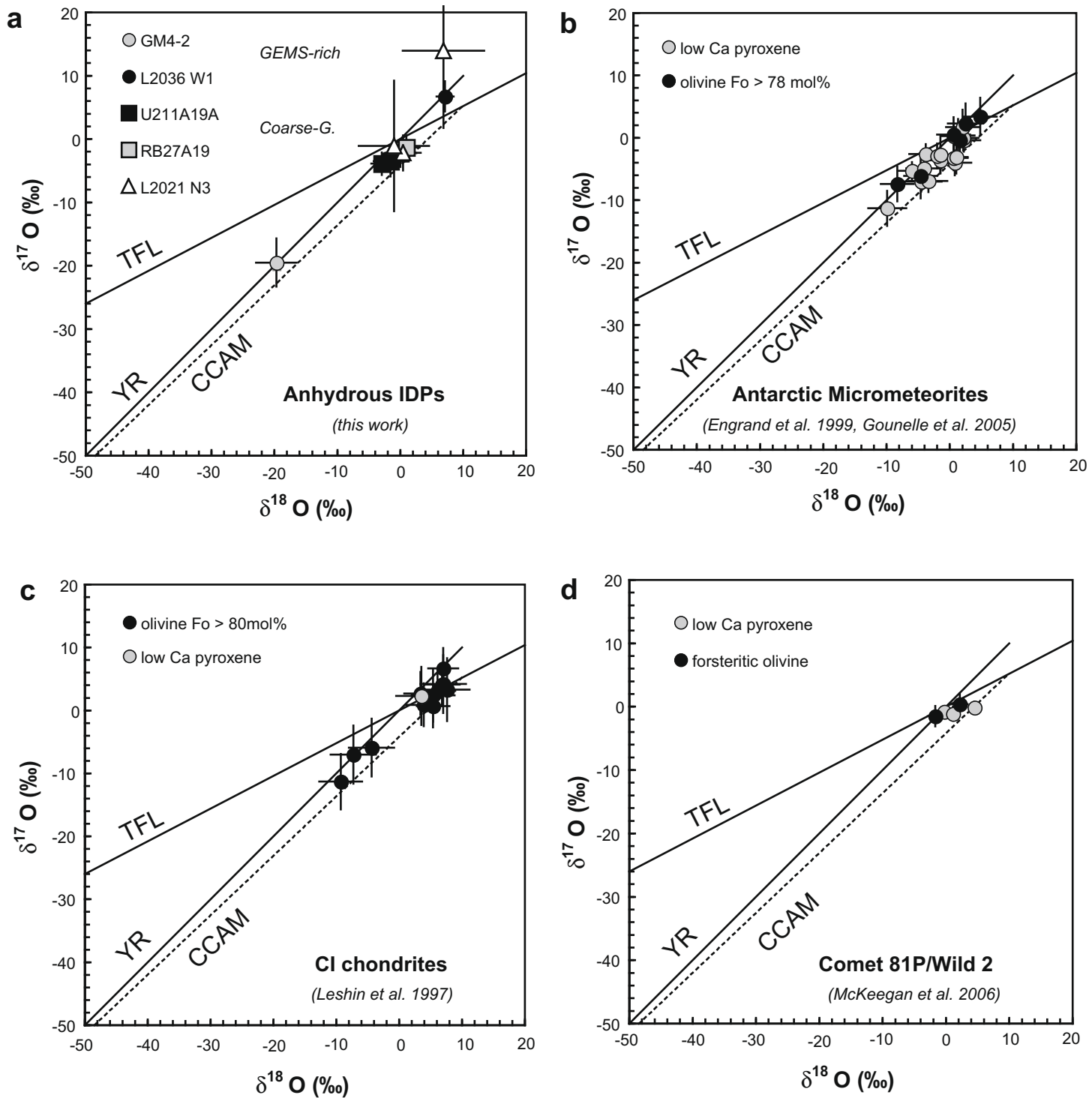

Fig. 5. O isotopes in anhydrous ferromagnesian components from putative or verified cometary samples. (a) Anhydrous IDPs. (b) Olivine and pyroxene in AMMs. (c) Olivine and pyroxene in Orgueil. (d) Olivine and pyroxene in comet Wild 2. Error bars are 2 $\sigma$. TFL stands for Terrestrial mass Fractionation Line, CCAM stands for Carbonaceous Chondrite Anhydrous Minerals mixing line and YR stands for Young and Russell slope 1.0 line (Young and Russell, 1998).

composition of the most primitive nebular components of IDPs to obtain new insights on early solar nebula processes.

\subsection{Parent-bodies of chondritic IDPs}

\subsubsection{Hydrated IDPs}

The observed spread in $\Delta^{17} \mathrm{O}(\sim 5 \%)$ in hydrated IDPs is comparable to that of individual ion probe analyses of hydrated carbonaceous chondrite matrices (e.g. Tagish Lake, Engrand et al., 2001; Leshin et al., 2001). The oxygen isotopic compositions of the hydrated IDPs studied here encompass those of all carbonaceous chondrites of petrologic type 1 and 2 (CI, Tagish Lake, CM and CR chondrites, Clayton and Mayeda, 1999; Brown et al., 2000, hereafter referred to as $\mathrm{C} 1 / \mathrm{C} 2$ chondrites). Thus, we can conclude that most hydrated IDPs come from parentbodies that underwent hydration under physico-chemical conditions (e.g. isotopic composition of the fluid, temperature, water-rock ratios) broadly comparable to those experienced by $\mathrm{C} 1 / \mathrm{C} 2$ parent-bodies.

In specific cases, oxygen isotopes are distinctive enough to classify individual IDPs as potentially related to particular meteorite groups, although in most cases oxygen isotopes are indicative only of an origin by similar processes rather than providing a definitive identification with a specific parent body. An example of the former case is given by IDP L2036R5 (Fig. 4a), an IDP dominated by hydrated silicates of CI-CM-like hydrogen isotopic composition (Aléon et al., 2001) and showing Fe-rich framboids common in $\mathrm{CI}$ and $\mathrm{CR}$ chondrites. This particle shows an 
$\mathrm{O}$ isotopic variability typical of $\mathrm{CI}$ chondrites in that regions dominated by Fe-rich framboids similar to magnetite have an $\mathrm{O}$ isotopic composition characteristic of magnetite in CI chondrites (Rowe et al., 1994), whereas regions dominated by $\mathrm{Mg}$-rich silicates have oxygen isotopic compositions comparable with those of CI phyllosilicates (Rowe et al., 1994). Together with $\mathrm{D} / \mathrm{H}$ isotope evidence and SEM observations, $\mathrm{O}$ isotopes strongly suggest that the parent body of L2036R 5 can be ascribed to CI carbonaceous chondrites.

In contrast, L2036B3 (Fig. 4b) has the heaviest bulk oxygen isotopic composition $\left(\delta^{18} \mathrm{O}=24.6 \pm 1.6 \%\right.$ measured in a chemically primitive (chondritic) object that was not appreciably heated (absence of textural evidence of atmospheric entry heating such as a compact molten matrix or vesicles). It is even heavier than the dehydrated carbonaceous chondrites B-7904, Y-82162 and the pair Y$86720 / Y-86789$ (hereafter $\mathrm{C} *$ chondrites) whose high $\delta^{18} \mathrm{O}$ values (21.07-22.29\%, Clayton and Mayeda, 1999) have been ascribed to metamorphism of CI-like chondrite material. The $\mathrm{O}$ isotopic composition of L2036B3 is comparable only to that of carbonates from hydrated carbonaceous chondrites (e.g. Leshin et al., 2001; Engrand et al., 2001; Benedix et al., 2003). Such high oxygen isotopic ratios could possibly be attributed to: (1) dehydration of CI-like phyllosilicates during thermal metamorphism in a $\mathrm{C} *$-like parent body, (2) a high abundance of minerals with large positive mineral-water fractionation factors such as carbonates, in an IDP from a CI-, CM- or Tagish Lake-like parent body, (3) formation of super-heavy phyllosilicates during aqueous alteration with a very large water-rock ratio in a carbonaceous chondrite parent body not represented in meteorite collections. In the absence of a detailed mineralogical characterization of the particle, none of these hypotheses can be ruled out. Matrajt et al. (2006) reported similar heavy $\mathrm{O}$ isotopic composition in seemingly unmelted AMMs. However, the description of their samples closely match that of scoriaceous AMMs (Genge et al., 2008), which are known to have been extensively heated. For instance, they report the presence of vesicles, which are commonly formed upon melt devolatilization during atmospheric entry (e.g. Toppani and Libourel, 2002). Furthermore, their $\mathrm{O}$ isotope systematics closely matches that of cosmic spherules (Engrand et al., 2005; Yada et al., 2005 ) with the most scoriaceous samples having the heaviest composition due to distillation during volatile loss. We note that, although an extensive atmospheric entry heating seems excluded for L2036B3, a moderate heating may have increased the extent of mass fractionation.

Finally, IDPs PP67 and U222C29 (Fig. 4c) have oxygen isotopic compositions closer to those of anhydrous meteorites and IDPs but are nevertheless indistinguishable from the matrices of CR and CM carbonaceous chondrites within error.

Most hydrated IDPs are not mineralogically identical to C1/C2 chondrites (e.g. Germani et al., 1990). Only in rare occasions have hydrated IDPs been identified as CM-type particles (Bradley and Brownlee, 1991; Rietmeijer, 1996) or CI-type particles (Keller et al., 1992). However, reflectance spectroscopy of hydrated IDPs indicates similarities with both $\mathrm{C} 1 / \mathrm{C} 2$ chondrites and main belt $\mathrm{C}$-type asteroids (Bradley et al., 1996). Our oxygen isotope data further suggest that hydrated IDPs come from parent-bodies that underwent hydration by water isotopically similar to the water that altered $\mathrm{C} 1 / \mathrm{C} 2$ meteorites.

\subsubsection{Anhydrous IDPs}

Like hydrated IDPs that have isotopic compositions comparable to those of bulk hydrated carbonaceous chondrites albeit over a larger range, anhydrous IDPs have isotopic compositions similar to bulk anhydrous carbonaceous chondrites but on a larger range. The oxygen isotopic composition of coarse-grained bulk IDPs and IDP minerals are very close to those of bulk $\mathrm{CV}, \mathrm{CO}$ and $\mathrm{CK}$ chondrites (Clayton and Mayeda, 1999). The spread along the slope 1 line is similar in amplitude to that observed in most Mg-rich anhydrous minerals in carbonaceous chondrites and micrometeorites (e.g. Weinbruch et al., 1993; Engrand et al., 1999a; Leshin et al., 2000) including those (precursor grains) in hydrated chondrites (Leshin et al., 1997, 2000, 2001; Engrand et al., 2001, Fig. 5).

Coarse-grained IDPs U211A19A and RB27A19, which may be petrographically similar to microchondrules (U211A19A shows an intergrowth of forsterite and enstatite) or individual matrix olivine (Mn-rich forsterite grains such as RB27A19 are commonly found isolated in carbonaceous chondrite matrices), could thus be attributed at first sight to matrix fragments of anhydrous carbonaceous chondrites. However, the study of the dust samples from comet Wild 2 has revealed the presence of similar coarse grains dominated by enstatite and forsterite with similar chondrule-like $\mathrm{O}$ isotopic composition (McKeegan et al., 2006). Microchondrule-like objects with textural, mineralogical and isotopic properties of chondrules in carbonaceous chondrites have also recently been found in Wild 2 samples (Nakamura et al., 2008). Their presence as well as that of a CAI in the Stardust samples has been attributed to a large scale mixing of high-temperature minerals formed in the inner solar system (McKeegan et al., 2006; Nakamura et al., 2008; Simon et al., 2008).

Unfortunately, $\mathrm{O}$ isotopic analysis of the most finegrained material from comet Wild 2 is hampered by severe contamination from the silica aerogel used as a collection medium. Fine-grained anhydrous IDPs and especially GEMS-rich IDPs have no petrographic equivalent in meteorite collections and have commonly been considered to be cometary dust particles (Bradley and Brownlee, 1986). Despite severe modifications during impact capture, the chemical compositions of anhydrous minerals in Stardust samples suggest that the dust from comet Wild 2 is more closely related to fine-grained anhydrous IDPs than any other extraterrestrial material (Zolensky et al., 2006) and possibly to friable Concordia AMMs (Duprat et al., 2007; Engrand et al., 2007; Dobrica et al., 2008). We note that only a few chondrites and IDPs have been analyzed at the scale of Wild 2 samples, which may introduce some bias in the comparison (Zolensky et al., 2008). The lack of GEMS and enstatite whiskers in Stardust samples is also interpreted by Ishii et al. (2008) as evidence that the rocky fraction of comet Wild 2 is more closely related to meteor- 
ites than chondritic porous anhydrous IDPs, which would then represent even more primitive cometary materials. Our O isotope data indicate that even "primitive" GEMS-rich IDPs of probable cometary origin are related to anhydrous carbonaceous chondrites.

Any distinction between coarse-grained IDPs, on the one hand, and highly porous fine-grained anhydrous IDPs on the other, and inferences regarding their respective parent-bodies may thus not be so obvious: indeed the coarsegrained IDPs U211A19A and RB27A19 are encased in a matrix of fine-grained material comparable to fine-grained IDPs (Steele, 1990). In the light of Stardust data, the oxygen isotopic compositions of anhydrous IDPs suggest that cometary dust is made of aggregates of fine-grained and coarse-grained particles both having oxygen isotopic compositions similar to those of anhydrous components of carbonaceous chondrites (anhydrous minerals in $\mathrm{C} 1 / \mathrm{C} 2$ chondrites and fractions or bulk $\mathrm{C} 3 / \mathrm{C} 4$ chondrites) and micrometeorites (Engrand et al., 1999a).

To summarize, the oxygen isotopic compositions of anhydrous IDPs are similar to those of bulk anhydrous carbonaceous chondrites (CV, CO, CK and ungrouped type 3 chondrites such as Acfer 094) and their dominant anhydrous minerals. Importantly, $\mathrm{O}$ isotopes in anhydrous IDPs are also similar to oxygen in the components of hydrated meteorites that escaped aqueous alteration, including the anhydrous minerals in Orgueil, as well as the anhydrous minerals in AMMs and comet Wild 2 (Fig. 5). Although, with the exception of the latter, the parent-body sources of most of these materials are not known with certainty, few would argue against an asteroidal origin of the chondrites (although a cometary origin for Orgueil has been proposed, Gounelle et al., 2006), and both asteroidal and cometary origins have been suggested for AMMs (Engrand and Maurette, 1998; Genge and Grady, 2002; Genge, 2006). The oxygen isotopic data thus suggest that anhydrous carbonaceous chondrites (CCs), hydrated CCs, AMMs, comet Wild 2 and IDPs were made of the same type of material prior to any hydration: primary solar system dust. Although they have no petrographic equivalent in meteorite collections, it seems likely that anhydrous IDPs probably sample many objects from a related group of small solar system objects of carbonaceous chondrite affinity which is distinct from ordinary, enstatite, K- or R-chondrite asteroids. This family would include both the asteroidal anhydrous CCs and comet Wild 2. The presence of microchondrules with properties akin to those of carbonaceous chondrite chondrules in comet Wild 2 (Nakamura et al., 2008) supports this inference. Thus comet Wild 2 may be considered as a type of carbonaceous chondrite. Anhydrous IDPs may represent the best preserved material that we have in our collections from these cometary parentbodies or their closest relatives.

\subsection{Relationship between hydrated and anhydrous chondritic IDPs}

We have shown that the rough sorting of chondritic IDPs into two classes of objects related to hydrated carbonaceous chondrites (hydrated IDPs) and to anhydrous carbonaceous chondrites and comet Wild 2 (anhydrous IDPs) agrees with a classification via $\mathrm{O}$ isotopic properties. The oxygen isotopic composition of hydrated carbonaceous chondrites is well accounted for by hydration of initially anhydrous carbonaceous chondrite asteroids during aqueous circulations on the parent body (e.g. Leshin et al., 1997; Clayton and Mayeda, 1999; Young et al., 1999). Here, we develop a similar model that extends the range of possible precursors to anhydrous IDPs. We consider to what extent the $\mathrm{O}$ isotopic compositions of hydrated IDPs can be explained by hydration of such precursors, that is to evaluate if the bulk CCs hydration model can be extended at the $10 \mu \mathrm{m}$ scale.

We used the framework of the simple hydration model of Clayton and Mayeda $(1984,1999)$ to investigate the isotopic effect of hydration on protosolar dust or dust aggregates similar to anhydrous IDPs. It is assumed that: (1) the starting material has the oxygen isotopic composition of the anhydrous IDPs as determined from this study, (2) the starting water has the composition of typical hydration water from type 1 and 2 carbonaceous chondrites (reservoir $\mathrm{HW}$ in Clayton and Mayeda, 1999 with $\delta^{17} \mathrm{O}=17.7 \%$, $\delta^{18} \mathrm{O}=28.1 \%$ and $\Delta^{17} \mathrm{O}=3.1 \%$ ), and (3) the temperature is $150^{\circ} \mathrm{C}$ as taken by Clayton and Mayeda, 1999 for the maximum temperature for alteration on CI chondrites. We did not use the isotopic composition of nebular water as inferred from the Acfer 094 cosmic symplectite (Sakamoto et al., 2007) since it is so different from that of hydrated IDPs that the model would obviously result in phyllosilicates with $\mathrm{O}$ isotopic composition differing from those we measured by at least several tens of permil.

This model must be considered as a first order approximation since it is based on the formation of serpentine, which is abundant in CI and CM chondrites (Zolensky and McSween, 1988) but is not so abundant in hydrated IDPs, which are often dominated by smectites (Germani et al., 1990). In addition, the alteration indexes, determined from the isotopic difference between whole rock and matrix separated fraction (Clayton and Mayeda, 1999) and used to estimate the progress of the reaction, are unavailable for IDPs. In spite of the good agreement between isotopic alteration indexes and petrographic alteration indexes in CM chondrites (Browning et al., 1996; Clayton and Mayeda, 1999), using petrographic alteration indexes in IDPs would require a detailed mineralogical study of the present IDPs, which is not available.

As expected, the model (Fig. 6) shows that the O isotopic compositions of all hydrated IDPs can be obtained by hydration of anhydrous IDPs. Hydration models of the ${ }^{16} \mathrm{O}$-poor and ${ }^{16} \mathrm{O}$-rich GEMS-rich IDPs (L2036W1 and GM4-2, respectively) provide envelope models bracketing all isotopic compositions of hydrated IDPs, suggesting that the former broadly represent the range of starting compositions, while most data points are close to those predicted by hydration of a parent body with the average isotopic composition of anhydrous IDPs $\left(\delta^{17} \mathrm{O}=-2.21 \%, \quad \delta^{18} \mathrm{O}=\right.$ $0.45 \%$ and $\Delta^{17} \mathrm{O}=-2.44 \%$ ) which is close to bulk anhydrous carbonaceous chondrites. Isotopic compositions of Orgueil and Tagish Lake are also in good agreement with this model. Using a lower temperature of $\sim 50{ }^{\circ} \mathrm{C}$ (Leshin 


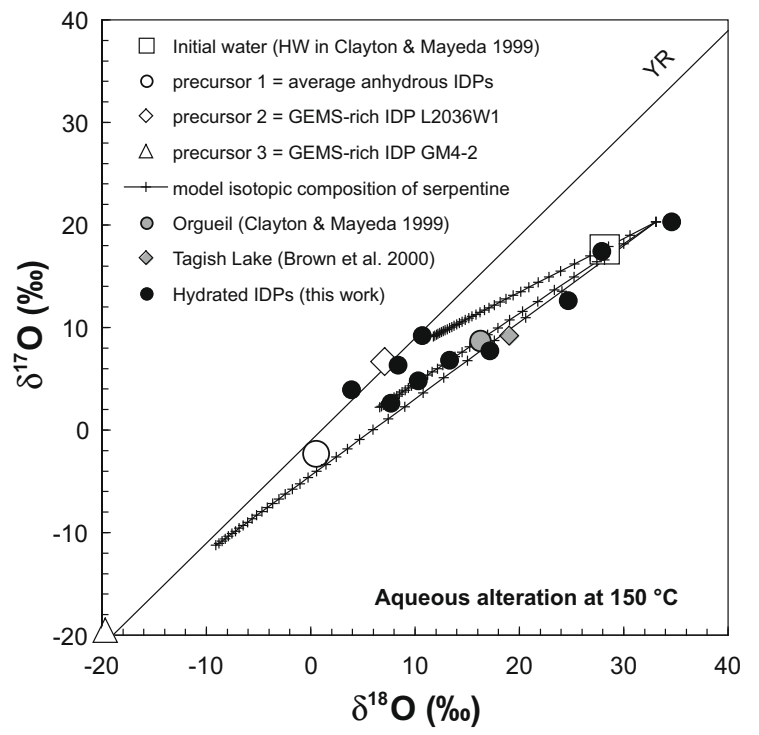

Fig. 6. Hydration model of anhydrous IDPs. Oxygen isotopic composition of serpentine produced by alteration of olivine is shown for three different precursor composition (average anhydrous IDPs and the two GEMS-rich IDPs) and encompasses the composition of all hydrated IDPs. Bulk composition of Orgueil and Tagish Lake are shown for comparison. Errors are not shown for clarity. YR stands for Young and Russell slope 1.0 line (Young and Russell, 1998).

et al., 1997) would result in an even larger range of possible compositions and would result in a similar conclusion. The $\sim 10 \mu \mathrm{m}$ scale of our analyses indicates that the hydration model of carbonaceous chondrite objects developed for bulk samples remains valid at a much smaller scale.

This type of model is useful to investigate the hydration history and hydration conditions of individual IDPs. The O isotopic compositions of hydrated IDPs close to those of anhydrous IDPs (e.g. PP67) could either reflect abundance of anhydrous minerals (e.g. Ca-Al-rich grains?) or hydration with water/rock ratios close to the minimum required by the model $(\sim 0.29)$. For comparison, the average water/rock ratio determined from $\mathrm{CM}$ chondrites (for which oxygen isotopes compare well with those of these IDPs, Fig. 4) is approximately 0.5 (Clayton and Mayeda, 1999). In contrast, the isotopic compositions heavier than those of CI chondrites may reflect extremely large water/ rock ratio ( $\mathrm{O}$ atoms in water relative to $\mathrm{O}$ atoms in rock) under comparable alteration conditions (temperature, duration and extent of reaction) or lower temperature with comparable water/rock ratios. This ratio is estimated to be $>2.7$ in Orgueil (Clayton and Mayeda, 1999) and may be up to 4-5 (e.g. in L2036B3) assuming that the heavy oxygen isotopic compositions are not due to later dehydration during thermal metamorphism.

Detailed mineralogical studies at the sub- $\mu \mathrm{m}$ scale indicate that the reality is more complex than this simple model. Indeed, anhydrous minerals are not rare in hydrated IDPs: e.g. forsterite, enstatite or diopside (e.g. Germani et al., 1990) and glass or GEMS can be present (Bradley, 1988; Nakamura et al., 2005a). So-called "tar balls" were described in hydrated IDPs, which are now recognized to be GEMS since they consist of chondritic glasses with embedded metal and sulfides and various amount of carbonaceous materials (see Bradley, 1988). Similarly, a wide variety of hydrated minerals (various phyllosilicates such as smectite, illite, kaolinite, mica-related phyllosilicates or carbonates) have been reported as minor components of dominantly anhydrous IDPs (Rietmeijer, 1991). This mixing of hydrated and anhydrous minerals at the nanoscale suggests that the progress of the hydration reactions is not complete in IDPs in agreement with the survival of GEMS in hydrothermal alteration experiments of anhydrous IDPs (Nakamura et al., 2005b).

The results of this model reinforce the previous suggestion that carbonaceous chondrites (both hydrated and anhydrous), the parent-bodies of AMMs and IDPs (both anhydrous and hydrated) and comet Wild 2 are all genetically related, in that they initially accreted dust from the same batches of well-mixed nebular reservoirs before any subsequent hydration or metamorphism. Oxygen isotope compositions thus seem to support a chemical continuum between carbonaceous chondrite asteroids (both hydrated and anhydrous) and comets. Because both hydrated and anhydrous objects are found among carbonaceous chondrites, chondritic IDPs and, based on a comparison of Wild 2 vs. Tempel 1, also comets (e.g. Lisse et al., 2006), the degree of hydration is probably not a good indicator of an asteroidal vs. a cometary origin.

This suggestion of a compositional continuum between inner solar system (i.e. asteroid belt) objects, such as carbonaceous chondrites, and outer solar system (i.e. Kuiper belt) objects, such as comets, is supported by several lines of evidence arising from astronomical observations and modeling. For example, models of the dynamical evolution of asteroid and comet populations show that asteroids can exist in comet-like orbits, including possibly up to $2.3 \%$ of the Oort cloud population (Weissman and Levison, 1997). In addition, recent observations suggest that dormant/ extinct comets possibly represent up to $8 \%$ of Near Earth Objects (DeMeo and Binzel, 2008). Secondly, remote sensing indicates several compositional similarities between some asteroids and some comets, e.g. asteroids rich in water ice (Thomas et al., 2005; Marchis et al., 2006) and comets with albedos similar to D-type asteroids (Licandro et al., 2003; Abell et al., 2005). Observations indicate similar silicate mineralogy between Trojan asteroids and comet HaleBopp (Emery et al., 2006). Additionally, some objects, such as 4015/Wilson-Harrington and 2060/Chiron, long considered to be asteroids have subsequently been discovered to have faint or irregular cometary activity (Weissman et al., 2002), and a population of main belt comets has now been identified (Hsieh and Jewitt, 2006). It is notable that the reflectance spectra of anhydrous chondritic IDPs as well as the Tagish Lake carbonaceous chondrite, whose parent body is a possible source of some hydrated micrometeorites (Nozaki et al., 2006), indicate similarities with P- and Dtype asteroids (Bradley et al., 1996; Hiroi et al., 2001). To what extent this similarity can be extended to Kuiper Belt Objects discovered in the last 10 years remains to be investigated. Thus, the petrographic and isotopic properties of 
chondritic interplanetary dust particles and micrometeorites likely indicates the sampling of various outer solar system bodies having preserved to a certain extent unprocessed material from the early solar nebula.

\subsection{Anhydrous IDPs and $O$ isotope reservoirs in the early solar system}

The mineralogy and chemistry of anhydrous IDPs indicates that they have escaped thermal and aqueous processing since their agglomeration in the protosolar nebula. In that respect, their components are likely to be either presolar interstellar dust grains or among the first dust particles that were made in the solar system. The small isotopic range among the five anhydrous IDPs studied here and their individual components (forsterite, enstatite, GEMSrich areas, Table 1) suggests that the sub-components of anhydrous IDPs have a similarly restricted range of isotopic composition, although a larger database would be required to better assess this conclusion. This composition is closer to most planetary materials than any other astronomical object: it is different from that of the CAIs (e.g. McKeegan and Leshin, 2001), probably from that of the Sun (Hashizume and Chaussidon, 2005; McKeegan et al., 2009) and from any nucleosynthetic components, whether of stellar (e.g. Clayton and Nittler, 2004) or non-thermal nucleosynthetic origin (i.e. irradiation, Aléon et al., 2005). In agreement with the recent results from the Stardust mission (McKeegan et al., 2006; Nakamura et al., 2008), this indicates that comets or comet-related objects are made of typical solar system material rather than being aggregates of unprocessed interstellar grains (e.g. Greenberg, 1982, 1998).

Mineralogical studies of anhydrous IDPs (e.g. Bradley and Brownlee, 1986; Bradley et al., 1989; Bradley, 1994; Keller et al., 2000; Keller and Messenger, 2005) have shown that they are aggregates of: (1) directly condensed crystalline grains, (2) amorphous grains condensed in non-equilibrium conditions, (3) thermally annealed grains, or (4) grains rendered amorphous upon prolonged irradiation. Depending on their formation, anhydrous IDP constituents could thus have preserved the initial isotopic composition of the gas from which they condensed or they could have been equilibrated with a gas of different $\mathrm{O}$ isotopic composition during thermal events/irradiation. They may also have preserved isotopic effects due to irradiation.

Among these processes, the efficiency of isotope exchange between a gaseous reservoir and porous chondritic amorphous grains still remains to be evaluated. In addition, the relative efficiencies of isotope exchange at moderately high temperature $(\sim 1000 \mathrm{~K})$ versus thermal annealing cannot be established because annealing data are mostly available for nanometer-sized smokes having properties controlled by surface energies (Fabian et al., 2000), whereas isotope exchange data are only available for micrometer-sized and larger particles, in which properties are controlled by volume energies. Isotopic exchange in crystalline particles, by contrast, can be evaluated using self-diffusion kinetics of oxygen in olivine (ol, Gerard and Jaoul, 1989) and clinopyroxene (cpx, Ryerson and McKeegan, 1994). We present calculations done for $500 \mathrm{~nm}$ spherical particles (Fig. 7) assuming that 90\% equilibration is reached. At temperatures $\sim 1000 \mathrm{~K}$ thought to characterize silicate annealing, more than $10^{6}$ and $10^{9}$ years are required to equilibrate clinopyroxene and olivine, respectively, with the gas. Even at $1500 \mathrm{~K}$, outside the range of any realistic temperature estimates, the equilibration times become $\sim 150 \mathrm{~h}$ for clinopyroxene and $\sim 80$ years for olivine. These calculations indicate that crystalline dust grains in the protosolar nebula retain their original isotopic composition, and if they are condensates, this must be the same as the composition of the gas from which they condensed.

LIME forsterite grains, such as those measured in RB27A19, are usually thought to be direct condensates from the nebular gas (Klöck et al., 1989). Similarly, isolated euhedral forsterite crystals such as that measured in L2021N3 can be produced by direct condensation from the nebular gas (Toppani et al., 2006). Therefore, the close-to-planetary isotopic composition of these grains is likely to be the composition of the nebular gas when and/ or where these grains condensed. This relatively ${ }^{16} \mathrm{O}$-poor composition is unlike that of ${ }^{16} \mathrm{O}$-rich forsterite in Amoeboid Olivine Aggregates (e.g. Aléon et al., 2002; Fagan et al., 2004) thought to have condensed in the innermost nebula (Krot et al., 2002) but is comparable to that of the nebular gas in which chondrules formed (Chaussidon et al., 2008). If this is correct it would imply that LIME forsterites condensed in a different environment than AOAs, perhaps at a greater heliocentric distance, but may have sampled a similar environment than that in which chondrules were melted.

The IDP U211A19A has a texture reminiscent of a microchondrule (Steele, 1990) and an oxygen isotopic composition typical of a chondrule. This IDP is thus

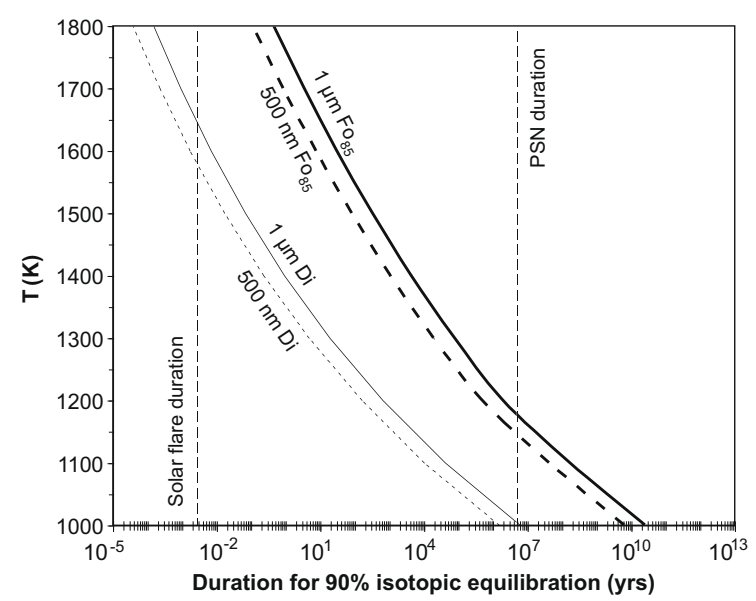

Fig. 7. Diffusion of $\mathrm{O}$ in sub- $\mu \mathrm{m}$ crystalline olivine $(85 \mathrm{~mol} \%$ forsterite, $\mathrm{Fo}_{85}$, Gerard and Jaoul, 1989) and pyroxene grains (diopside, Di, Ryerson and McKeegan, 1994). The temperature of heating is shown as a function of the duration required to reach $90 \%$ isotopic equilibrium, i.e. when the gas and solids are not different by more than a few $\%$. Unless strongly heated at temperatures typical of the CAI-forming regions in the innermost nebula, the diffusion of oxygen is too slow to achieve isotopic equilibrium during the lifetime of the solar nebula. 
petrographically and isotopically akin to the microchondrules found in comet Wild 2 (Nakamura et al., 2008). Since it was also associated with sub- $\mu \mathrm{m}$ chondritic porous material before sectioning (Steele, 1990), it may represent the first cometary chondrule identified in IDP collections.

Whether they are chondrule fragments or condensates, the typical planetary $\mathrm{O}$ isotopic composition of forsterite and enstatite crystals measured in anhydrous IDPs and comet Wild 2 (McKeegan et al., 2006; Nakamura et al., 2008, this work) strongly suggest that crystalline forsterite and enstatite detected by mid-infrared spectroscopy in comets (e.g. Crovisier et al., 1997) and circumstellar disks (e.g. Malfait et al., 1998; Van Boekel et al., 2004; Kessler-Silacci et al., 2006) are indeed formed in the inner part of the accretion disk rather than being annealed interstellar grains (e.g. Bockelee-Morvan et al., 2002).

It has been argued that GEMS are interstellar dust grains that underwent exposure to particle irradiation in the interstellar medium (Bradley, 1994). However, recent evidence indicates that most GEMS have $\mathrm{O}$ isotopic composition within $10 \%$ of that of the solar system (Stadermann and Bradley, 2003; Keller and Messenger, 2005), which led to the suggestion that many GEMS could be non-equilibrium condensates from the protosolar nebula gas (Keller and Messenger, 2005). Experiments performed under reducing conditions suggest that GEMS could also be totally amorphous interstellar grains that acquired their final mineralogical properties upon annealing in the protosolar nebula (Davoisne et al., 2006). The two GEMS-rich IDPs analyzed in this study show the largest range of ${ }^{16} \mathrm{O}$ variations among anhydrous chondritic IDPs, which suggests that GEMS could potentially be distributed along a slope 1 line with various ${ }^{16} \mathrm{O}$ excesses and depletions, in the range of other planetary materials.

We have begun a systematic high precision analysis of more GEMS-rich IDPs to confirm this observation and establish the full range of $\mathrm{O}$ isotopic compositions of GEMS-rich particles. It is important to note that individual GEMS units may distribute along a substantially greater range on a slope 1 line than the limited data presented here would indicate. Indeed, the isotopic analysis of individual GEMS $\sim 100 \mathrm{~nm}$ in size is physically limited by the number of $\mathrm{O}$ atoms. One should appreciate that even in an idealized mass spectrometer having a $100 \%$ useful yield, the analysis of a $100 \mathrm{~nm}$ GEMS would result in a $1 \sigma$ uncertainty of $\sim 10 \%$ on $\delta^{17} \mathrm{O}$. For grains below $100 \mathrm{~nm}$ in diameter this uncertainty rapidly reaches the full range of $\mathrm{O}$ isotopic compositions measured in the solar system. In a more realistic mass spectrometer with a useful yield of $\sim 10 \%$ (e.g. IMS 1270, NanoSIMS), the $1 \sigma$ uncertainty from Poisson statistics is still $\sim 15 \%$ for a $\sim 200 \mathrm{~nm}$ grain.

Although our limited data do not allow a clear conclusion, we suggest that GEMS may have recorded physico-chemical processes resulting in mass-independent fractionation, such as mixing of reservoirs (dust-gas) during condensation or partial isotopic exchange with the solar gas during annealing. Such a trend is unlikely to result from preservation of presolar nucleosynthetic signatures or equilibration with the interstellar gas during sputter-deposition processes, since both would result in a broader scattering around the solar value rather than a dependence on the ${ }^{16} \mathrm{O}$ content only.

If further analyses confirm the planetary-like (i.e. relatively ${ }^{16} \mathrm{O}$-poor) composition of GEMS that our data suggest, then this would pose severe problem for reconciling an interstellar origin of GEMS with isotopic self-shielding of $\mathrm{CO}$ as the origin of oxygen isotope variations in the solar system. All the self-shielding models require an ${ }^{16} \mathrm{O}$-rich composition for the precursor interstellar dust, close to that of the Sun (Hashizume and Chaussidon, 2005; McKeegan et al., 2009). Thus, if GEMS are really representative of amorphous dust grains from the presolar cloud (or even the outer solar system), then another mechanism may be required to explain the isotopic disequilibrium between such primordial materials and the bulk solar system as represented by the Sun. On the other hand, if GEMS were formed in the inner solar system, they must have acquired their $\mathrm{O}$ isotopic compositions which are comparable to those of chondrules, even though they have a totally different thermal history from anhydrous materials processed at high temperature. Because GEMS anneal above $900 \mathrm{~K}$ (Brownlee et al., 2005), they clearly escaped whatever thermal events formed chondrules, whether shock wave melting of dust (e.g. Desch and Connolly, 2002) or planetesimal fragmentation (Libourel and Krot, 2007). Thus they must have formed at a different time or in a different locale.

\section{CONCLUSION}

Oxygen isotopic abundances were measured in nine chondritic IDPs. All IDPs, including the mineralogically pristine porous, anhydrous, GEMS-rich IDPs, have typical planetary-like compositions, close to terrestrial and chondritic values. Large isotopic anomalies indicative of abundant presolar interstellar dust grains have not been found. Hydrated IDPs have $\mathrm{O}$ isotopic compositions suggesting that their parent-bodies are related to hydrated $\mathrm{C} 1 / \mathrm{C} 2$ carbonaceous chondrites. Anhydrous IDPs exhibit variations in their ${ }^{16} \mathrm{O}$ content as observed in anhydrous carbonaceous chondrites and in comet Wild 2. As in carbonaceous chondrites, hydrated IDPs can be derived from anhydrous IDPs by aqueous alteration. Oxygen isotopes thus suggest that asteroidal carbonaceous chondrites, the parent-bodies of chondritic IDPs, of most AMMs and comet Wild 2 all belong to the same family of small bodies of carbonaceous chondrite affinity.

The oxygen isotopic compositions of crystalline olivine and pyroxene from anhydrous IDPs are close to those of the nebular gas that interacted with chondrule melts and are similar to mafic minerals in comet Wild 2 suggesting that these minerals were formed in inner nebular regions. Together with their mineralogical properties, the isotopic data suggest that forsterite and enstatite detected in comets and at large orbital distances in other stellar systems were probably formed in the inner disk rather than being annealed interstellar grains. Two GEMS-rich IDPs are the most ${ }^{16} \mathrm{O}$-rich and ${ }^{16} \mathrm{O}$-depleted among the IDPs studied thus far but are still within $20 \%$ of the terrestrial composition, suggesting that GEMS may not be interstellar dust grains or that their $\mathrm{O}$ isotopic composition is not correctly accounted for by 
self-shielding models. More analyses are required to establish if this variation in the ${ }^{16} \mathrm{O}$ content is representative.

\section{ACKNOWLEDGEMENTS}

We would like to thank John Bradley and Don Brownlee for providing the IDPs studied in 1999 and the curation team at the Johnson Space Center for providing the IDPs studied in 2005. Ian Hutcheon is warmly thanked for hosting one of us (JA) during the 2005 session. Together with JEOL, he also provided necessary help with the brand new LLNL FEG-SEM. All the ion probe group in Nancy is warmly thanked for discussions, help and maintenance of the IMS 1270 during the 2003-2004 "small beam analysis" development sessions. Alice Aléon-Toppani is thanked for numerous discussions about the nature of GEMS. Advice from Rick Ryerson and Mike Toplis about oxygen self-diffusion is appreciated. We would like to thank in-depth revision and advices from the associate editor Christian Koeberl, from Matthew Genge and two other anonymous reviewers. The UCLA ion probe laboratory is partially supported by the NSF Instrumentation and Facilities program. This work was supported by a grant from the NASA Cosmochemistry program and by the LLNL Institute of Geophysics and Planetary Physics. This work was performed under the auspices of the US Department of Energy by the Lawrence Livermore National Laboratory under Contract DE-AC52-07NA27344.

\section{REFERENCES}

Abell P. A., Fernandez Y. R., Pravec P., French L. M., Farnham T. L., Gaffey M. J., Hardersen P. S., Kusnirak P., Sarounova L., Sheppard S. S. and Narayan G. (2005) Physical characteristics of comet nucleus $\mathrm{C} / 2001 \mathrm{OG}_{108}$ (LONEOS). Icarus 179, 174-194.

Aléon J., Engrand C., Robert F. and Chaussidon M. (2001) Clues on the origin of interplanetary dust particles from the isotopic study of their hydrogen-bearing phases. Geochim. Cosmochim. Acta 65, 4399-4412.

Aléon J., Krot A. N. and McKeegan K. D. (2002) Calciumaluminum-rich inclusions and amoeboid olivine aggregates from the CR carbonaceous chondrites. Meteorit. Planet. Sci. 37, 1729-1755.

Aléon J., Robert F., Chaussidon M. and Marty B. (2003) Nitrogen isotopic composition of macromolecular organic matter in interplanetary dust particles. Geochim. Cosmochim. Acta 67, 3773-3783.

Aléon J., Robert F., Duprat J. and Derenne S. (2005) Extreme oxygen isotope ratios in the early Solar System. Nature 437, 385-388.

Aléon J., McKeegan K. D. and Leshin L. (2006) Oxygen isotopes in chondritic interplanetary dust: parent-bodies and nebular oxygen reservoirs. Lunar Planet. Sci. XXXVII. \#1921 (abstr.) (CD-ROM).

Amelin Y., Krot A. N., Hutcheon I. D. and Ulyanov A. A. (2002) Lead isotopic ages of chondrules and calcium-aluminum-rich inclusions. Science 297, 1678-1683.

Benedix G. K., Leshin L. A., Farquhar J., Jackson T. and Thiemens M. H. (2003) Carbonates in CM2 chondrites: constraints on alteration conditions from oxygen isotopic compositions and petrographic observations. Geochim. Cosmochim. Acta 67, 1577-1588.

Bockelee-Morvan D., Gautier D., Hersant F., Huré J.-M. and Robert F. (2002) Turbulent radial mixing in the solar nebula as the source of crystalline silicates in comets. Astron. Astrophys. 384, 1107-1118.
Bradley J. P. (1988) Analysis of chondritic interplanetary dust thinsections. Geochim. Cosmochim. Acta 52, 889-900.

Bradley J. P. (1994) Chemically anomalous preaccretionally irradiated grains in interplanetary dust from comets. Science 265, 925-929.

Bradley J. P. and Brownlee D. E. (1986) Cometary particles - thin sectioning and electron beam analysis. Science 231, 1542-1544.

Bradley J. P., Brownlee D. E. and Germani M. S. (1989) Automated thin-film analyses of anhydrous interplanetary dust particles in the analytical electron microscope. Earth Planet. Sci. Lett. 93, 1-13.

Bradley J. P. and Brownlee D. E. (1991) An interplanetary dust particle linked directly to type-CM meteorites and an asteroidal origin. Science 251, 549-552.

Bradley J. P., Humecki H. J. and Germani M. S. (1992) Combined infrared and analytical electron microscope studies of interplanetary dust particles. Astrophys. J. 394, 643-651.

Bradley J. P., Keller L. P., Brownlee D. E. and Thomas K. L. (1996) Reflectance spectroscopy of interplanetary dust particles. Meteorit. Planet. Sci. 31, 394-402.

Brown P. G., Hildebrand A. R., Zolensky M. E., Grady M., Clayton R. N., Mayeda T. K., Tagliaferri E., Spalding R., MacRae N. D., Hoffman E. L., Mittlefehldt D. W., Wacker J. F., Bird J. A., Campbell M. D., Carpenter R., Gingerich H., Glatiotis M., Greiner E., Mazur M. J., JA McCauslandP., Plotkin H. and Rubak Mazur T. (2000) The fall, recovery, orbit, and composition of the Tagish Lake meteorite: a new type of carbonaceous chondrite. Science 290, 320-325.

Browning L. B., McSween, Jr., H. Y. and Zolensky M. E. (1996) Correlated alteration effects in $\mathrm{CM}$ carbonaceous chondrites. Geochim. Cosmochim. Acta 60, 2621-2633.

Brownlee D. E. (1985) Cosmic dust: collection and research. Ann. Rev. Earth Planet. Sci. 13, 147-173.

Brownlee D. E., Joswiak D. J., Bradley J. P., Matrajt G. and Wooden D. H. (2005) Cooked GEMS - insights into the hot origin of crystalline silicates in circumstellar disks and the cold origin GEMS. Lunar Planet Sci. XXXVI. \#2391 (CDROM).

Brownlee D. and 182 co-authors (2006) Comet 81P/Wild 2 under a microscope. Science 314, 1711-1716.

Chaussidon M., Libourel G. and Krot A. N. (2008) Oxygen isotopic constraints on the origin of magnesian chondrules and on the gaseous reservoirs in the early solar system. Geochim. Cosmochim. Acta 72, 1924-1938.

Choi B. G., McKeegan K. D., Krot A. N. and Wasson J. T. (1998) Extreme oxygen-isotope compositions in magnetite from unequilibrated ordinary chondrites. Nature 392, 577-579.

Clayton D. D. and Nittler L. R. (2004) Astrophysics with presolar stardust. Ann. Rev. Astron. Astrophys. 42, 39-78.

Clayton R. N. (2002) Self shielding in the solar nebula. Nature 415, 860-861.

Clayton R. N. and Mayeda T. K. (1984) The oxygen isotope record in Murchison and other carbonaceous chondrites. Earth Planet. Sci. Lett. 67, 151-161.

Clayton R. N. and Mayeda T. K. (1996) Oxygen isotope studies of achondrites. Geochim. Cosmochim. Acta 60, 1999-2017.

Clayton R. N. and Mayeda T. K. (1999) Oxygen isotope studies of carbonaceous chondrites. Geochim. Cosmochim. Acta 63, 20892104.

Clayton R. N., Grossman L. and Mayeda T. K. (1973) A component of primitive nuclear composition in carbonaceous meteorites. Science 182, 485-488.

Clayton R. N., Onuma N., Grossman L. and Mayeda T. K. (1977) Distribution of the pre-solar components in Allende and other carbonaceous chondrites. Earth Planet. Sci. Lett. 34, 209-224. 
Clayton R. N., Mayeda T. K., Goswami J. N. and Olsen E. J. (1991) Oxygen isotope studies of ordinary chondrites. Geochim. Cosmochim. Acta 55, 2317-2337.

Crovisier J., Leech K., Bockelee-Morvan D., Brooke T. Y., Hanner M. S., Altieri B., Keller H. U. and Lellouch E. (1997) The spectrum of comet Hale-Bopp (C/1995 O1) observed with the Infrared Space Observatory at 2.9 astronomical units from the Sun. Science 275, 1904-1907.

Davoisne C., Djouadi Z., Leroux H., D’Hendecourt L., Jones A. and Deboffle D. (2006) The origin of GEMS in IDPs as deduced from microstructural evolution of amorphous silicates with annealing. Astron. Astrophys. 448, L1-L4.

DeMeo F. and Binzel R. P. (2008) Comets in the Near-Earth Object population. Icarus 194, 436-449.

Desch S. J. and Connolly, Jr., H. C. (2002) A model of the thermal processing of particles in solar nebula shocks: application to the cooling rates of chondrules. Meteorit. Planet. Sci. 37, 183-207.

Dobrica E., Engrand C., Leroux H., Duprat J. and Gounelle M. (2008) Classic and exotic particles in the 2006 Concordia Antarctic micrometeorite collection. Lunar Planet. Sci. XXXIX. \#1672 (CD-ROM).

Duprat J., Engrand C., Maurette M., Kurat G., Gounelle M. and Hammer C. (2007) Micrometeorites from Central Antarctic snow: The CONCORDIA collection. Adv. Space Res. 39, 605-611.

Emery J. P., Cruikshank D. P. and Van Cleve J. (2006) Thermal emission spectroscopy $(5.2-38 \mu \mathrm{m})$ of three Trojan asteroids with the Spitzer space telescope: detection of fine-grained silicates. Icarus 182, 496-512.

Engrand C. and Maurette M. (1998) Carbonaceous micrometeorites from Antarctica. Meteorit. Planet. Sci. 33, 565-580.

Engrand C., McKeegan K. D. and Leshin L. A. (1999a) Oxygen isotopic compositions of individual minerals in Antarctic micrometeorites: further links to carbonaceous chondrites. Geochim. Cosmochim. Acta 63, 2623-2636.

Engrand C., McKeegan K. D., Leshin L. A., Bradley J. P. and Brownlee D. E. (1999b) Oxygen isotopic compositions of interplanetary dust particles: ${ }^{16} \mathrm{O}$-excess in a GEMS-rich IDP. Lunar Planet. Sci. XXX. \#1690 (abstr.) (CD-ROM).

Engrand C., Gounelle M., Duprat J. and Zolensky M. E. (2001) Insitu oxygen isotopic composition of individual minerals in Tagish Lake, a new type 2 carbonaceous meteorite. Lunar Planet. Sci. XXXII. \#1568 (CD-ROM).

Engrand C., McKeegan K. D., Leshin L. A., Herzog G. F., Schnabel C., Nyquist L. and Brownlee D. E. (2005) Isotopic compositions of oxygen, iron, chromium and nickel in cosmic spherules: toward a better comprehension of atmospheric entry heating effects. Geochim. Cosmochim. Acta 69, 5365-5385.

Engrand C., Duprat J., Maurette M. and Gounelle M. (2007) Fe$\mathrm{Ni}$ sulfides in Concordia Antarctic micrometeorites. Lunar Planet. Sci. XXXVIII. \#1668 (abstr.) (CD-ROM).

Fabian D., Jäger C., Henning Th., Dorschner J. and Mutschke H. (2000) Steps toward interstellar silicate mineralogy V. Thermal evolution of amorphous magnesium silicates and silica. Astron. Astrophys. 364, 282-292.

Fagan T. J., Krot A. N., Keil K. and Yurimoto H. (2004) Oxygen isotopic evolution of amoeboid olivine aggregates in the reduced CV3 chondrites Efremovka, Vigarano, and Leoville. Geochim. Cosmochim. Acta 68, 2591-2611.

Feuchtgruber H., Lellouch E., Bézard B., Encrenaz T., de Graauw T. and Davis G. R. (1999) Detection of HD in the atmospheres of Uranus and Neptune: a new determination of the $\mathrm{D} / \mathrm{H}$ ratio. Astron. Astrophys. 341, L17-L21.

Floss C., Stadermann F. J., Bradley J., Dai Z. R., Bajt S. and Graham G. (2004) Carbon and nitrogen isotopic anomalies in an anhydrous interplanetary dust particle. Science 303, 13551358.
Fraundorf P. (1981) Interplanetary dust in the transmission electron microscope: diverse materials from the early solar system. Geochim. Cosmochim. Acta 45, 915-943.

Genge M. J. (2006) Ordinary chondrite micrometeorites from the Koronis asteroids. Lunar Planet. Sci. XXXVII. \#1759 (CDROM).

Genge M. J. and Grady M. M. (2002) The distribution of asteroids: evidence from Antarctic micrometeorites. Lunar Planet. Sci. XXXIII. \#1010 (CD-ROM).

Genge M. J., Engrand C., Gounelle M. and Taylor S. (2008) The classification of micrometeorites. Meteorit. Planet. Sci. 43, 497515.

Gerard O. and Jaoul O. (1989) Oxygen diffusion in San Carlos olivine. J. Geophys. Res. 94, 4119-4128.

Germani M. S., Bradley J. P. and Brownlee D. E. (1990) Automated thin film analyses of hydrated interplanetary dust particles in the analytical microscope. Earth Planet. Sci. Lett. 101, 162-179.

Gounelle M., Engrand C., Maurette M., Kurat G., McKeegan K. D. and Brandstätter F. (2005) Small Antarctic micrometeorites $(25-50 \mu \mathrm{m})$ : a mineralogical and in situ oxygen isotopic study. Meteorit. Planet. Sci. 40, 917-932.

Gounelle M., Spurný P. and Bland P. A. (2006) The orbit and atmospheric trajectory of the Orgueil meteorite from historical records. Meteorit. Planet. Sci. 41, 135-150.

Greenberg J. M. (1982) What are comets made of - a model based on interstellar dust. In Comets (ed. L. L. Wilkening). University of Arizona Press, Tucson, pp. 131-163.

Greenberg J. M. (1998) Making a comet nucleus. Astron. Astrophys. 330, 375-380.

Grossman L. (1972) Condensation in the primitive solar nebula. Geochim. Cosmochim. Acta 36, 597-619.

Hashizume K. and Chaussidon M. (2005) A non-terrestrial ${ }^{16} \mathrm{O}$ rich isotopic composition for the protosolar nebula. Nature $\mathbf{4 3 4}$, 619-622.

Hashizume K. and Chaussidon M. (2008) Evidence of at least two extra-selenial components accreting on the moon - in search for the oxygen isotopic composition of the solar component trapped in lunar metallic grains. Lunar Planet. Sci. XXXIX. \#1391 (abstr.) (CD-ROM).

Hiroi T., Zolensky M. E. and Pieters C. M. (2001) The Tagish Lake meteorite: a possible sample from a D-type asteroid. Science 293, 2234-2236.

Hsieh H. H. and Jewitt D. (2006) A population of comets in the main asteroid belt. Science 312, 561-563.

Ireland T. R., Holden P., Norman M. D. and Clarke J. (2006) Isotopic enhancements of ${ }^{17} \mathrm{O}$ and ${ }^{18} \mathrm{O}$ from solar wind particles in the lunar regolith. Nature 440, 776-778.

Ishii H. A., Bradley J. P., Dai Z. R., Chi M., Kearsley A. T., Burchell M. J., Browning N. D. and Molster F. (2008) Comparison of comet $81 \mathrm{P} /$ Wild 2 dust with interplanetary dust from comets. Science 319, 447.

Jedwab J. (1971) La magnétite de la météorite d'Orgueil vue au microscope électronique à balayage. Icarus 15, 319-340.

Keller L. P. and Messenger S. (2005) The nature and origin of interplanetary dust: high temperature components. In Chondrite and the Protoplanetary disks (eds. A. N. Krot, E. R. D. Scott and B. Reipurth). ASP conf. series, vol. 341. San Francisco, pp. 657-665.

Keller L. P., Thomas K. L. and McKay D. S. (1992) An interplanetary dust particle with links to CI chondrites. Geochim. Cosmochim; Acta 56, 1409-1412.

Keller L. P., Messenger S. and Bradley J. P. (2000) Analysis of a deuterium-rich interplanetary dust particle (IDP) and implications for presolar material in IDPs. J. Geophys. Res. 105, 10397-10402. 
Kessler-Silacci J., Augereau J.-C., Dullemond C. P., Geers V., Lahuis F., Evans, II, N. J., van Dishoeck E. F., Blake G. A., Adwin Boogert A. C., Brown J., Jorgensen J. K., Knez C. and Pontoppidan K. M. (2006) C2d SPITZER IRS spectra of disks around T Tauri stars. I. Silicate emission and grain growth. Astrophys. J. 639, 275-291.

Klöck W., Thomas K. L., McKay D. S. and Palme H. (1989) Unusual olivine and pyroxene composition in interplanetary dust and unequilibrated ordinary chondrite. Nature 339, 126-128.

Krot A. N., McKeegan K. D., Leshin L. A., MacPherson G. J. and Scott E. R. D. (2002) Existence of an ${ }^{16} \mathrm{O}$-rich gaseous reservoir in the solar nebula. Science 295, 1051-1054.

Lellouch E., Bézard B., Fouchet T., Feuchtgruber H., Encrenaz T. and de Graauw T. (2001) The deuterium abundance in Jupiter and Saturn from ISO-SWS observations. Astron. Astrophys. 370, 610-622.

Leshin L. A., Rubin A. E. and McKeegan K. D. (1997) The oxygen isotopic composition of olivine and pyroxene from CI chondrites. Geochim. Cosmochim. Acta 61, 835-845.

Leshin L. A., McKeegan K. D. and Benedix G. K. (2000) Oxygen isotope geochemistry of olivine from carbonaceous chondrites. Lunar Planet. Sci. XXXI. \#1918 (abstr.) (CD-ROM).

Leshin L. A., Farquhar J., Guan Y., Pizzarello S., Jackson T. L. and Thiemens M. H. (2001) Oxygen isotopic anatomy of Tagish Lake: relationship to primary and secondary minerals in CI and CM chondrites. Lunar Planet. Sci. XXXII. \#1843 (abstr.) (CDROM).

Libourel G. and Krot A. N. (2007) Evidence for the presence of planetesimal material among the precursors of magnesian chondrules of nebular origin. Earth Planet. Sci. Lett. 254, 1-8.

Licandro J., Campins H., Hergenrother C. and Lara L. M. (2003) Near-infrared spectroscopy of the nucleus of comet 124P/ Mrkos. Astron. Astrophys. 398, L45-L48.

Lisse C. M., VanCleve J., Adams A. C., A'Hearn M. F., Fernandez Y. R., Farnham T. L., Armus L., Grillmair C. J., Ingalls J., Belton M. J. S., Groussin O., McFadden L. A., Meech K. J., Schultz P. H., Clark B. C., Feaga L. M. and Sunshine J. M. (2006) Spitzer spectral observations of the Deep Impact ejecta. Science 313, 635-640.

Lyons J. R. and Young E. D. (2005) CO self-shielding as the origin of oxygen isotope anomalies in the early solar nebula. Nature 435, 317-320.

Malfait K., Waelkens C., Waters L. B. F. M., Vandenbussche B., Huygen E. and de Graauw M. S. (1998) The spectrum of the young star HD 100546 observed with the Infrared Space Observatory. Astron. Astrophys. 332, L25-L28.

Marchis F., Hestroffer D., Descamps P., Berthier J., Bouchez A. H., Campbell R. D., Chin J. C. Y., van Dam M. A., Hartman S. K., Johansson E. M., Lafon R. E., Le Mignant D., de Pater I., Stomski P. J., Summers D. M., Vachier F., Wizinovich P. L. and Wong M. H. (2006) A low density of $0.8 \mathrm{~g} \mathrm{~cm}^{-3}$ for the Trojan binary asteroid 617 Patroclus. Nature 439, 565-567.

Marcus R. A. (2004) Mass-independent isotope effect in the earliest processed solids in the solar system: a possible chemical mechanism. J. Chem. Phys. 121, 8201-8211.

Matrajt G., Guan Y., Leshin L., Taylor S., Genge M., Joswiak D. and Brownlee D. (2006) Oxygen isotope measurements of individual unmelted Antarctic micrometeorites. Geochim. Cosmochim. Acta 70, 4007-4018.

Maurette M., Olinger C., Christophe M., Kurat G., Pourchet M., Brandstätter F. and Bourot-Denise M. (1991) A collection of diverse micrometeorites recovered from 100 tons of Antarctic blue ice. Nature 351, 44-47.

McKeegan K. D. (1987) Oxygen isotopes in refractory stratospheric dust particles: proof of extraterrestrial origin. Science 237, 1468-1471.
McKeegan K. D. and Leshin L. A. (2001) Stable isotope variations in extraterrestrial materials. In Stable Isotope Geochemistry (eds. J. W. Valley and D. R. Cole). Mineralogical Society of America, Washington, pp. 279-318.

McKeegan K. D., Aléon J., Bradley J., Brownlee D., Busemann H., Butterworth A., Chaussidon M., Fallon S., Floss C., Gilmour J., Gounelle M., Graham G., Guan Y., Heck P. R., Hoppe P., Hutcheon I. D., Huth J., Ishii H., Ito M., Jacobsen S. B., Kearsley A., Leshin L. A., Liu M.-C., Lyon I., Marhas K., Marty B., Matrajt G., Meibom A., Messenger S., Mostefaoui S., Mukhopadhyay S., Nakamura-Messenger K., Nittler L., Palma R., Pepin R. O., Papanastassiou D. A., Robert F., Schlutter D., Snead C. J., Stadermann F. J., Stroud R., Tsou P., Westphal A., Young E. D., Ziegler K., Zimmermann L. and Zinner E. (2006) Isotopic compositions of cometary matter returned by stardust. Science 314, 1724-1728.

McKeegan K. D., Kallio A. P., Heber V., Jarzebinski G., Mao P. H., Coath C. D., Kunihiro T., Wiens R., Allton J. and Burnett D. S. (2009) Oxygen isotopes in a Genesis concentrator sample. Lunar Planet. Sci. XL. \#2494 (abstr.) (CD-ROM).

Messenger S., Keller L. P., Stadermann F. J., Walker R. M. and Zinner E. (2003) Samples of stars beyond the solar system: silicate grains in interplanetary dust. Science 300, 105-108.

Nakamura K., Messenger S. and Keller L. P. (2005a) TEM and Nanosims study of hydrated/anhydrous phase mixed IDPs: cometary or asteroidal origin? Lunar Planet. Sci. XXXVI. \#1824 (CD-ROM).

Nakamura K., Messenger S. and Keller L. P. (2005b) Experimental hydrothermal alteration of anhydrous IDPs. Meteorit. Planet. Sci. 40, A110.

Nakamura T., Noguchi T., Tsuchiyama A., Ushikubo T., Kita N. T., Valley J. W., Zolensky M. E., Kakazu Y., Sakamoto K., Mashio E., Uesugi K. and Nakano T. (2008) Chondrulelike objkects in short-period comet 81P/Wild 2. Science 321, 1664 1667.

Nozaki W., Nakamura T. and Noguchi T. (2006) Bulk mineralogical changes of hydrous micrometeorites during heating in the upper atmosphere at temperature below $1000^{\circ} \mathrm{C}$. Meteorit. Planet. Sci. 41, 1095-1114.

Rietmeijer F. J. M. (1991) Aqueous alteration in five chondritic porous interplanetary dust particles. Earth Planet. Sci. Lett. 102, 148-157.

Rietmeijer F. J. (1996) CM-like interplanetary dust particles in lower stratosphere during 1989 October and 1991 June/July. Meteorit. Planet. Sci. 31, 278-288.

Robert F. (2004) The common property of isotopic anomalies in meteorites. Astron. Astrophys. 415, 1167-1176.

Rowe M. W., Clayton R. N. and Mayeda T. K. (1994) Oxygen isotopes in separated components of CI and CM meteorites. Geochim. Cosmochim. Acta 58, 5341-5347.

Ryerson F. J. and McKeegan K. D. (1994) Determination of oxygen self-diffusion in åkermanite, anorthite, diopside, and spinel: implications for oxygen isotopic anomalies and the thermal histories of $\mathrm{Ca}-\mathrm{Al}$-rich inclusions. Geochim. Cosmochim. Acta 58, 3713-3734.

Sakamoto N., Seto Y., Itoh S., Kuramoto K., Fujino K., Nagashima K., Krot A. N. and Yurimoto H. (2007) Remnants of the early solar system water enriched in heavy oxygen isotopes. Science 317, 231-233.

Seto Y., Sakamoto N., Fujino K., Kaito T., Oikawa T. and Yurimoto H. (2008) Mineralogical characterization of a unique material having heavy oxygen isotope anomaly in matrix of the primitive carbonanceous chondrite Acfer 094. Geochim. Cosmochim. Acta 72, 2723-2734.

Simon S. B., Joswiak D. J., Ishii H. A., Bradley J. P., Chi M., Grossman L., Aléon J., Brownlee D. E., Fallon S., Hutcheon I. 
D., Matrajt G. and McKeegan K. D. () A refractory inclusion returned by Stardust from Comet P81/Wild2. Meteorit. Planet. Sci. 43, 1861-1877.

Stadermann, F. J.; Bradley, J. P. (2003) The isotopic nature of GEMS in interplanetary dust particles. Meteorit. Planet. Sci. 38. \#5236 (abstr.).

Steele I. M. (1990) Minor elements in forsterites of Orgueil (C1), Alais $(\mathrm{C} 1)$ and two interplanetary dust particles compared to C2-C3-UOC forsterites. Meteoritics 25, 301-307.

Thiemens M. H. (1999) Mass-independent isotope effects in planetary atmospheres and the early solar system. Science 283, 341-345.

Thiemens M. H. and Heidenreich, III, J. E. (1983) The massindependent fractionation of oxygen: a novel isotope effect and its possible cosmochemical implications. Science 219, 10731075.

Thomas K. L., Blanford G. E., Clemett S. J., Flynn G. J., Keller L. P., Klock W., Maechling C. R., McKay D. S., Messenger S., Nier A. O., Schlutter D. J., Sutton S. R., Warren J. L. and Zare R. N. (1995) An asteroidal breccia: the anatomy of a cluster IDP. Geochim. Cosmochim. Acta 59, 2797-2815.

Thomas P. C., Parker J. Wm., McFadden L. A., Russell C. T., Stern S. A., Wykes M. V. and Young E. F. (2005) Differentiation of the asteroid Ceres as revealed by its shape. Nature 437, 224-226.

Toppani A. and Libourel L. (2002) Experimental study of micrometeorite vesiculation. Lunar Planet Sci. XXXIII. \#1473 (CD-ROM).

Toppani A., Libourel G., Engrand C. and Maurette M. (2001) Experimental simulation of atmospheric entry of micrometeorites. Meteorit. Planet. Sci. 36, 1377-1396.

Toppani A., Libourel G., Robert F. and Ghanbaja J. (2006) Laboratory condensation of refractory dust in protosolar and circumstellar conditions. Geochim. Cosmochim. Acta 70, 50355060.

Van Boekel R., Min M., Leinert Ch., Waters L. B. F. M., Richichi A., Chesneau O., Dominik C., Jaffe W., Dutrey A., Graser U., Henning Th., de Jong J., Köhler R., de Koter A., Lopez B., Malbet F., Morel S., Paresce F., Perrin G., Preibisch Th., Przygodda F., Schöller M. and Wittkowski M. (2004) The building blocks of planets within the 'terrestrial' region of protoplanetary disks. Nature 432, 479-482.

Weinbruch S., Zinner E. K., El Goresy A., Steele I. M. and Palme H. (1993) Oxygen isotopic composition of individual olivine grains from the Allende meteorite. Geochim. Cosmochim. Acta 57, 2649-2661.

Weissman P. R. and Levison H. F. (1997) Origin and evolution of the unusual object 1996 PW: asteroids from the Oort cloud. Astrophys. J. 488, L133-L136.

Weissman P. R., Bottke, Jr., W. F. and Levison H. F. (2002) Evolution of comets into asteroids. In Asteroids III (eds. W. F.
Bottke Jr., A. Cellino, P. Paolicchi and R. P. Binzel). University of Arizona Press, Tucson, pp. 669-686.

Yada T., Nakamura T., Noguchi T., Matsumoto N., Kusakabe M., Hiyagon H., Ushikubo T., Sugiura N., Kojima H. and Takaoka N. (2005) Oxygen isotopic and chemical compositions of cosmic spherules collected from the Antarctic ice sheet: implications for their precursor materials. Geochim. Cosmochim. Acta 69, 5789-5804.

Young E. D. and Russell S. S. (1998) Oxygen reservoirs in the early solar nebula inferred from an Allende CAI. Science 282, 452455.

Young E. D., Ash R. D., England P. and Rumble, III, D. (1999) Fluid flow in chondritic parent-bodies: deciphering the compositions of planetesimals. Science 286, 1331-1335.

Yurimoto H. and Kuramoto K. (2004) Molecular cloud origin for the oxygen isotope heterogeneity in the solar system. Science 305, 1763-1766.

Zolensky M. and McSween, Jr., H. Y. (1988) Aqueous alteration. In Meteorites and the Early Solar System (eds. J. F. K. Kerridge and M. S. Matthews). University of Arizona Press, Tucson, pp. 114-143.

Zolensky M. E., Zega T. J., Yano H., Wirick S., Westphal A. J., Weisberg M. K., Weber I., Warren J. L., Velbel M. A., Tsuchiyama A., Tsou P., Toppani A., Tomioka N., Tomeoka K., Teslich N., Taheri M., Susini J., Stroud R., Stephan T., Stadermann F. J., Snead C. J., Simon S. B., Simionovici A., See T. H., Robert F., Rietmeijer F. J. M., Rao W., Perronnet M. C., Papanastassiou D. A., Okudaira K., Ohsumi K., Ohnishi I., Nakamura-Messenger K., Nakamura T., Mostefaoui S., Mikouchi T., Meibom A., Matrajt G., Marcus M. A., Leroux H., Lemelle L., Le L., Lanzirotti A., Langenhorst F., Krot A. N., Keller L. P., Kearsley A. T., Joswiak D., Jacob D., Ishii H., Harvey R., Hagiya K., Grossman L., Grossman J. N., Graham G. A., Gounelle M., Gillet P., Genge M. J., Flynn G., Ferroir T., Fallon S., Ebel D. S., Dai Z. R., Cordier P., Clark B., Chi M., Butterworth A. L., Brownlee D. E., Bridges J. C., Brennan S., Brearley A., Bradley J. P., Bleuet P., Bland P. A. and Bastien R. (2006) Mineralogy and petrology of comet 81P/Wild 2 nucleus samples. Science 314, 1735-1739.

Zolensky M., Nakamura-Messenger K., Rietmeijer F., Leroux H., Mikouchi T., Ohsumi K., Simon S., Grossman L., Stephan T., Weisberg M., Velbel M., Zega T., Stroud R., Tomeoka K., Ohnishi I., Tomioka N., Nakamura T., Matrajt G., Joswiak D., Brownlee D., Langenhorst F., Krot A., Kearsley A., Ishii H., Graham G., Dai Z. R., Chi M., Bradley J., Hagiya K., Gounelle M., Keller L. and Bridges J. (2008) Comparing Wild 2 particles to chondrites and IDPs. Meteorit. Planet. Sci. 43, 261272.

Associate editor: Christian Koeberl 